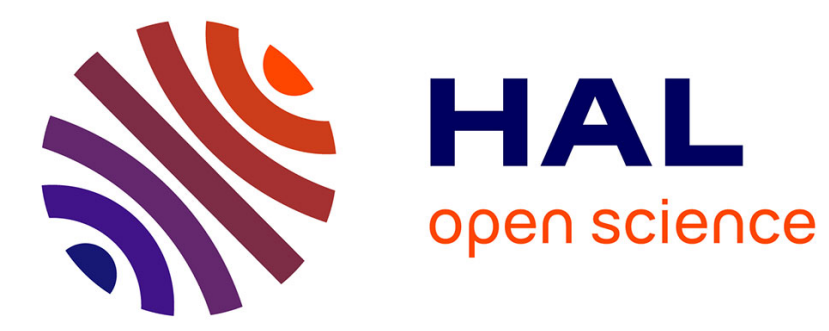

\title{
Apport et réception française de la Charte européenne des langues régionales ou minoritaires: approche sociolinguistique
}

Alain Viaut

\section{- To cite this version:}

Alain Viaut. Apport et réception française de la Charte européenne des langues régionales ou minoritaires: approche sociolinguistique. Revue d'Etudes Comparatives Est-Ouest, 2002, 33 (1), pp.9-48. 10.3406/receo.2002.3131 . halshs-01411579

\section{HAL Id: halshs-01411579 \\ https://shs.hal.science/halshs-01411579}

Submitted on 7 Dec 2016

HAL is a multi-disciplinary open access archive for the deposit and dissemination of scientific research documents, whether they are published or not. The documents may come from teaching and research institutions in France or abroad, or from public or private research centers.
L'archive ouverte pluridisciplinaire HAL, est destinée au dépôt et à la diffusion de documents scientifiques de niveau recherche, publiés ou non, émanant des établissements d'enseignement et de recherche français ou étrangers, des laboratoires publics ou privés. 


\section{Apport et réception française de la Charte européenne des langues régionales ou minoritaires : approche sociolinguistique}

In: Revue d'études comparatives Est-Ouest. Volume 33, 2002, №1. Dossier: Points de vue sur le Charte européenne des langues régionales ou minoritaires. pp. 9-48.

Citer ce document / Cite this document :

Viaut André. Apport et réception française de la Charte européenne des langues régionales ou minoritaires : approche sociolinguistique. In: Revue d'études comparatives Est-Ouest. Volume 33, 2002, N¹. Dossier: Points de vue sur le Charte européenne des langues régionales ou minoritaires. pp. 9-48.

doi : $10.3406 /$ receo.2002.3131

http://www.persee.fr/web/revues/home/prescript/article/receo_0338-0599_2002_num_33_1_3131 


\section{Abstract}

The European Charter for Regional or Minority Languages is an international convention proposed, in 1992, for signature to countries belonging to the Council of Europe. Work on drawing it up started in the 1980s, and it went into force as of 1998. After this rather long nascent phase, the Charter has met with success. Several major countries, especially in west Europe, have signed it. France, one of the few exceptions, has based its refusal on a quite strict official and public monolingualism. As the only international convention specialized in this field, the Charter covers neither the languages of migrants nor dialects. It aims to protect regional and minority languages whether or not they correspond to specific territories. The conditions for signing the Charter are flexible: in relation to a hundred or so clauses with an incompressible base (the preamble and sections I and II), ratification is valid if 35 clauses are accepted. As a result of this Charter, a European standard for protecting and fostering regional and minority languages in the private and public spheres is taking shape.

\section{Résumé}

La Charte européenne des langues régionales ou minoritaires est une convention internationale qui a été proposée à l'adhésion des États membres du Conseil de l'Europe en 1992. Son élaboration remontait au début des années 1980 et elle ne put commencer à être mise en application qu'à partir de 1998. Après cette phase d'émergence un peu longue, elle connaît maintenant du succès depuis que de nombreux grands pays, notamment d'Europe occidentale, y ont adhéré, la France étant un des seuls à ne pas l'avoir fait en s'appuyant sur des conceptions de monolinguisme officiel et public très strict. Seul texte international à être aussi spécialisé dans ce domaine, la Charte ne concerne cependant ni les langues de migrants ni les dialectes. Elle a pour but la protection des langues régionales ou minoritaires sans que des territoires leur correspondent forcément. Les conditions d'adhésion sont souples puisque, par rapport à une centaine de dispositions et sur une base incompressible minimale (préambule et parties I et II), la ratification peut être validée à partir de 35 dispositions souscrites. Le résultat déjà observable est celui d'un standard européen en train de se dégager en matière de protection et de promotion de ces langues dans la vie privée et publique.

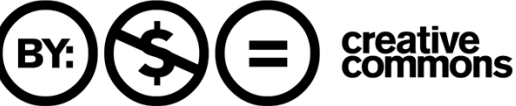




\title{
APPORT et RÉCEPTIOn fRAnÇAise de la Charte EUROPÉENNE DES LANGUES RÉGIONALES OU MINORITAIRES : APPROCHE SOCIOLINGUISTIQUE
}

\author{
Alain VIAUT *
}

\begin{abstract}
RÉSUMÉ : La Charte curopéenne des langues régionales ou minoritaires est une convention internationale qui a été proposée à l'adhésion des États membres du Conseil de l'Europe en 1992. Son élaboration remontait au début des années 1980 et elle ne put commencer à être mise en application qu'à partir de 1998. Après cette phase d'émergence un peu longue, elle connaît maintenant du succès depuis que de nombreux grands pays, notamment d'Europe occidentale, y ont adhéré, la France étant un des seuls à ne pas l'avoir fait en s'appuyant sur des conceptions de monolinguisme officiel et public très strict. Seul texte international à être aussi spécialisé dans ce domaine, la Charte ne concerne cependant ni les langues de migrants ni les dialectes. Elle a pour but la protection des langues régionales ou minoritaires sans que des territoires leur correspondent forcément. Les conditions d'adhésion sont souples puisque, par rapport à une centaine de dispositions et sur une base incompressible minimale (préambule et parties I et II), la ratification peut être validée à partir de 35 dispositions souscrites. Le résultat déjà observable est celui d'un standard européen en train de se dégager en matière de protection et de promotion de ces langues dans la vie privée et publique.
\end{abstract}

ABSTRACT : The European Charter for Regional or Minority Languages is an international convention proposed, in 1992, for signature to countries belonging to the Council of Europe. Work on drawing it up started in the 1980s, and it went into force as of 1998. After this rather long nascent phase, the Charter has met with success. Several major countries, especially in west Europe, have signed it. France, one of the few exceptions, has based its refusal on a quite strict official and public monolingualism. As the only international convention specialized in this field, the Charter covers neither the languages of migrants nor dialects. It aims to protect regional and minority languages whether or not they correspond to specific territories. The conditions for signing the Charter are flexible: in relation to a hundred or so clauses with an incompressible base (the preamble and sections I and II), ratification is valid if 35 clauses are accepted. As a result of this Charter, a European standard for protecting and fostering regional and minority languages in the private and public spheres is taking shape.

Le processus d'élaboration de la Charte européenne des langues régionales ou minoritaires a débuté dans les années quatre-vingt. Mise à la signature en 1992, elle s'avère adaptable aux différentes configurations linguistiques des pays membres d'un Conseil de l'Europe qui a vu, ces dernières années, sa base géographique s'étendre vers l'Est. Si ce texte ne propose pas forcément des

* Chargé de recherche au CNRS, rattaché à l'équipe Territorialité et identité dans le domaine curopéen (TIDE, UMR 6588 du CNRS), Université Michel de Montaigne Bordeaux III (e-mail : Alain.Viaut@msha.u-bordeaux.fr). 
mesures plus favorables que celles qui sont déjà en vigueur dans les États qui y ont adhéré ou se préparent à le faire, il contribue à les préciser, voire à les réajuster ou à les compléter et, dans tous les cas, à les consolider.

Parmi les questions auxquelles la Charte se propose de répondre, nous choisirons de porter notre attention sur celles qui concernent les sujets du droit et les usages linguistiques concernés par les mesures proposées, les types de langues « régionales ou minoritaires » susceptibles d'être visés par la Charte et leur perception en relation avec la notion de territoire. S'il sera fait davantage référence à la France qu'à d'autres pays européens dans ce propos, c'est en raison du débat de fond qui y est apparu lorsque la perspective de l'adhésion à la Charte s'y est présentée. C'est aussi parce que les prises de position officielles, jusque dans les oppositions apparues à leur sujet à l'échelon de l'exécutif, ont permis de vérifier autant l'adaptabilité de la Charte que la capacité d'un État à l'éluder. L'enjeu de ce qui peut aussi se passer dans d'autres pays n'est pas le sauvetage, la vie ou la survie de langues qui peuvent se maintenir ou être protégées sans cela. Pour notre part, nous utiliserons également ici, à côté des appellations institutionnalisées, la désignation de « langue peu répandue ». Nous la concevons comme englobante, aussi bien pour des langues minorées du fait de mesures ayant eu pour résultat de les marginaliser que pour des langues qui, sans être officielles d'un État, peuvent être répandues à l'intérieur d'une aire éventuellement restreinte et ne point pâtir d'un statut désavantageux.

La Charte ne saurait être une panacée et certains États ont déjà acquis sans elle une expérience éprouvée dans la gestion de leurs langues régionales ou minoritaires. En revanche, elle représente une garantie minimale de la mise en application d'un certain nombre de mesures connues et validécs, ćlaborées tant par des spécialistes que par des politiques, propres à assurer un aménagement linguistique de base ou plus développé, autrement dit un standard en la matière. La question sous-jacente que pose également la Charte est peut-être la suivante : veut-on faire le pari du fonctionnement du patrimoine culturel que représentent les langues régionales ou minoritaires en Europe ?

Dans l'étude qui suit, après avoir évoqué l'historique de ce texte, nous mettrons l'accent sur les différents moyens de cerner les objets linguistiques qui s'en dégagent en rapport avec la notion de territoire, puis nous aborderons les réactions émanant des États, la France en particulier, engagés dans les procédures d'adhésion ou les ayant accomplies, comme autant de premières mises en contexte de la Charte.

\section{1. ÉMERGENCE ET CARACTÉRISTIQUES DE LA CHARTE}

Sans détailler l'historique de la Charte, nous retiendrons d'abord que le projet d'émettre une législation internationale concernant les droits linguistiques en Europe n'est pas récent, contrairement à ce que certaines voix mal informées ont pu laisser entendre. Il n'est pas lié au règlement de conflits en cours ou poten- 
tiels depuis une dizaine d'années dans les régions balkanique et orientale de l'espace européen. On peut faire remonter les origines de la préparation d'un texte majeur destiné à aborder la question des contacts de langues hiérarchisés en Europe à la Recommandation 285 relative aux droits des minorités nationales, votée en 1961 par l'Assemblée parlementaire du Conseil de l'Europe. L'Assemblée demandait qu'un article concernant la protection des minorités nationales soit inclus dans le deuxième protocole additionnel à la Convention de sauvegarde des droits de l'homme et des libertés fondamentales de 1950, entrée en vigueur en $1953^{\prime}$. La formulation suivante fut proposée :

«Les personnes appartenant à une minorité nationale ne peuvent être privées du droit, en commun avec les autres membres de leur groupe et dans les limites assignées par l'ordre public, d'avoir leur propre vie culturelle, d'employer leur propre langue, d'ouvrir des écoles qui leur soient propres et de recevoir l'enseignement dans la langue de leur choix ou de professer et de pratiquer leur propre religion ».

En dépit de l'emploi des termes « groupe » et « minorité », il apparaissait que les sujets annoncés du droit étaient bien les « personnes », répondant en cela au texte initial de la Convention de 1950. Ni les langues minoritaires ni les groupes les utilisant n'étaient en fait désignés comme objets d'une protection. Cela fut évoqué à travers différents documents officiels. Ainsi, en 1981, la Recommandation 928 de l'Assemblée parlementaire du Conseil de l'Europe relative aux problèmes d'éducation et de culture posés par les langues minoritaires et les dialectes en Europe évoqua des mesures minimales de respect de l'identité linguistique dans la toponymie, de la langue maternelle des enfants à l'école, de son accès aux mass media et de la possibilité de l'utiliser «comme langue officielle ou co-officielle » dans les «territoires » concernés. Ce texte, adopté par l'Assemblée le 7 octobre, fut suivi et renforcé par la Résolution Arfé sur une charte communautaire des langues et cultures régionales et sur une charte des droits des minorités ethniques, votée le 16 octobre de la même année 1981 par le Parlement européen, cette fois, dans le cadre, plus restreint mais plus intégré, de la Communauté européenne. Le Parlement, « ayant pris acte de la vigoureuse résurgence de mouvements animés par des minorités ethniques et linguistiques qui souhaitent voir approfondir et reconnaître les raisons de leur identité historique » et interprétant cela comme « une source d'enrichissement » de la « civilisation européenne », invita son président à « transmettre la présente résolution au Conseil et à la Commission, aux gouvernements et aux autorités régionales des pays membres de la Communauté, ainsi qu'au Conseil de

1. L'article 14 de la Convention de sauvegarde des droits de l'homme et des libertés fondamentales évoquait déjà la langue au même titre que le sexe, la race ou la religion, parmi les droits et libertés devant être formellement protégés : « La jouissance des droits et libertés reconnus dans la présente Convention doit être assurée, sans distinction aucune, fondée notamment sur le sexe, la race, la couleur, la langue, la religion, les opinions politiques ou toutes autres opinions, l'origine nationale ou sociale, l'appartenance à une minorité nationale, la fortune, la naissance ou toute autre situation ». 
l'Europe ${ }^{2}$. La mise en relation des organes représentatifs de la Communauté européenne avec le Conseil de l'Europe sur la question des langues et des cultures régionales et sur celle des minorités ethniques, ainsi préconisée, sera rappelée par la suite, dans les mêmes termes, à la fin de la Résolution Kuijpers sur les langues et cultures des minorités régionales et ethniques de la Communauté européenne adoptée le 10 octobre 1987 par le Parlement européen. Ayant constaté que la Commission n'avait guère avancé sur ce sujet depuis la Résolution Arfé de 1981, « considérant qu'il subsiste de nombreux obstacles à l'exercice par les minorités nationales et linguistiques de leur spécificité culturelle et sociale et que les comportements à l'égard de ces minorités et de leurs problèmes restent souvent empreints de méconnaissance et d'incompréhension, voire, dans certains cas, de discrimination ${ }^{3}$, le Parlement européen annonça qu'il soutiendrait « les efforts déployés par le Conseil de l'Europe pour élaborer la Charte européenne des langues régionales ou minoritaires » et formula six recommandations détaillées. Il demanda en outre à la Commission « de conférer le rang d'organe consultatif officiel au Bureau européen des langues de moindre diffusion ». La Résolution Arfé avait été à l'origine de la création de ce bureau qui n'a cessé, depuis, d'agir en relation avec la Commission et le Parlement européen ainsi qu'avec le Conseil de l'Europe. Il apparut clairement, à partir de là, que l'élaboration d'un texte majeur, suggérée en 1981 par le Parlement européen dans l'intitulé même de la résolution préparée par Gaetano Arfé, était engagée dans le cadre du Conseil de l'Europe. De fait, cet organisme, depuis sa création en 1949, au lendemain de la Deuxième guerre mondiale, a fait de la sauvegarde et du développement des Droits de l'homme ${ }^{4}$ un de ses objectifs prioritaires. Ce processus déboucha en fin de compte sur la rédaction de deux conventions, répondant ainsi aux vœux formulés par le Parlement européen dans la Résolution Arfé de 1981. La Charte européenne des langues régionales ou minoritaires, désignée plus avant à l'aide de l'acronyme CELROM, qui sera adoptée le 25 juin 1992 à une large majorité du Comité des ministres du Conseil de l'Europe ${ }^{5}$ et ouverte à la signature le 5 novembre suivant, allait être le premier grand texte international entièrement consacré à la question des droits linguistiques et à la sauvegarde des langues peu répandues. La protection des minorités nationales, déjà abordée en revanche par plusieurs textes de cette nature, ferait l'objet d'une autre convention ouverte à la signature en février 1995 : la Convention-cadre pour la protection des minorités nationales.

Les contextes d'apparition de ces deux textes furent différents. À partir du début des années quatre-vingt, le Conseil de l'Europe spécialisa ses approches, tandis que le Parlement européen eut tendance à relier langues minoritaires et

2. Résolution (Arfé) sur une charte communautaire des langues et cultures régionales et sur une charte des droits des minorités ethniques (J.O. C 287 du 09.11.1981, p. 106).

3. Résolution (Kuijpers) sur les langues et cultures des minorités régionales et ethniques de la Communauté européenne (J.O. C 318 du 30.11.1987, p. 160).

4. Article 1, alinéa b du statut du Conseil de l'Europe.

5. Chypre, la France, le Royaume-Uni et la Turquie s'abstinrent. La Grèce vota contre et la Bulgarie fut absente lors de l'adoption. 
minorités nationales ou culturelles dans les Résolutions Kuijpers de 1987 et Killilea de 1994. La première se réfère aux « langues et cultures des minorités régionales et ethniques », la seconde aux «minorités linguistiques et culturelles ». Le contexte d'émergence de la Charte européenne des langues régionales ou minoritaires fut la résurgence de ces langues en Europe de l'Ouest à la fin des années soixante-dix. En particulier, la Recommandation 928 de l'Assemblée parlementaire, évoquée plus haut, et la Résolution 192 de 1988 (cf. infra) sur les langues régionales ou minoritaires prise par la Conférence permanente des pouvoirs locaux et régionaux de l'Europe (CPLRE), organisme dépendant du Conseil de l'Europe, chargé, avec un groupe d'experts indépendants et de politiques représentants l'Assemblée parlementaire, d'élaborer à partir de 1984 le texte de la future charte, abordèrent ce sujet en se référant aux autres textes internationaux et au cadre de l'Europe occidentale d'alors. Simplement, au moment où une première rédaction du texte qui avait été établie et adoptée par la CPLRE - il fut ensuite révisé à la baisse - fut présentée au Comité des ministres, il fut demandé d'en faire une convention et de la proposer à la signature des pays de l'Europe centrale et orientale qui n'adhéraient pas encore, à cette époque, au Conseil de l'Europe. La Convention-cadre fut, elle, conçue dans un autre contexte politique international. Élaborée au début des années quatre-vingt-dix, une des préoccupations de ses rédacteurs fut celle de participer au règlement des tensions et des conflits en Europe centrale et orientale. Une première proposition, datée du 8 février 1991, évoquait cette assise élargie de l'Europe dans un des considérants de son préambule : «Considérant qu'il existe des minorités dans les États membres du Conseil de l'Europe et dans les États d'Europe centrale et orientale ». Deux années plus tard, les chefs d'État et de gouvernement des pays membres du Conseil de l'Europe mirent l'accent, dans la Déclaration de Vienne du 9 octobre 1993, sur la situation dans l'exYougoslavie ${ }^{6}$, estimant « que la protection des minorités nationales est essentielle à la stabilité et à la sécurité démocratique de notre continent ». Il en découlait, à la fin de la Déclaration, un engagement à « rédiger à bref délai une convention-cadre précisant les principes que les États contractants s'engagent à respecter pour assurer la protection des minorités nationales ». Ce texte serait également ouvert à la signature des États non-membres, ceci pour pousser des pays de cette partie de l'Europe en proie à des changements à s'aligner sur les principes inscrits dans cette convention à venir. Elle fut effectivement mise au point peu après et mise à la signature le $1^{\text {er }}$ février 1995.

Ces quelques faits concernant cette dernière convention ne seraient guère importants dans le présent propos si celle-ci n'avait été associée au départ (cf. supra, Résolution Arfé) au projet qui déboucha sur la CELROM. Ils doivent également être mis en relation avec les confusions qui furent diffusées en France

6. «Nous condamnons tous ces égarements; ils plongent des peuples de l'ex-Yougoslavie dans la haine et dans la guerre et menacent d'autres régions. Nous appelons les dirigeants de ces peuples à mettre un terme à leurs conflits. Nous invitons ces peuples à nous rejoindre pour construire et consolider la nouvelle Europe". 
par des représentants de courants politiques ou idéologiques au nom d'une certaine conception de la laïcité et de la lutte contre l'obscurantisme et le fédéralisme européen rampant. Une des idées développées était, et continue parfois d'être, que la France ne saurait être concernée par une convention qui aurait été élaborée « dans le contexte de l'effondrement du Mur de Berlin et dans la perspective de l'adhésion des États d'Europe centrale et orientale au Conseil de l'Europe » (Combat lä̈que, 1999, p. 10). Selon ces opinions, elle ferait partie « d'un ensemble de traités visant à offrir une solution pacifique à la "question des nationalités", sur le modèle de l'ancien Empire austro-hongrois » (ibid.). Cela résulte d'informations erronées dans la mesure où les processus d'élaboration, comme nous l'avons vu, sont clairement situés dans le temps, et ce n'est pas la CELROM qui a été rédigée avec en toile de fond une situation préoccupante à l'est de l'Europe communautaire et de l'ensemble constitué par les pays membres du Conseil de l'Europe d'alors, mais bien la Convention-cadre pour la protection des minorités nationales. Au-delà de ces considérations d'origine polémique, si l'objet déclaré de la CELROM représente les langues régionales ou minoritaires d'Europe, la Convention-cadre s'intéresse aussi aux langues minoritaires dans la mesure où l'un des principaux traits identificateurs des minorités nationales est linguistique. Les articles 10 à 14, soit environ $15 \%$ de l'ensemble des articles déclinant les cinq titres de ce texte, lui sont consacrés avec, comme objet du droit, la personne, et, comme garantie de base, celle de l'usage tant privé que public des langues minoritaires. Elle préconise ainsi, comme le Parlement européen ou l'Assemblée parlementaire, certains principes premiers tels l'accès pour une langue minoritaire à un usage public ${ }^{7}$.

Si la France, en particulier, n'intègre pas dans son droit interne les groupes ou les minorités ${ }^{8}$, alors qu'il s'agit, essentiellement, dans la Convention-cadre d'en constater l'existence et de la gérer, force est de reconnaître que c'est, globalement, l'élaboration et l'adhésion à la CELROM qui auront posé le plus de difficultés. Les langues non officielles d'État soulèvent manifestement plus de problèmes pour être reconnues et gérées que d'autres caractéristiques culturelles

7. Cf. par exemple, en ce qui concerne le Parlement européen, l'article 1.c de la Résolution Arfé de 1981, l'article 6 de la Résolution Kuijpers de 1987, l'article 4 de la Résolution Killilea adoptée le 9 février 1994, ou bien, en ce qui concerne l'Organisation des Nations Unies, l'article 2 de la Déclaration des droits des personnes appartenant à des minorités nationales ou ethniques, religieuses et linguistiques, adoptée par l'Assemblée générale des Nations Unies le 18 décembre 1992, où, là, plutôt qu'un accès à l'administration publique, comme dans la Résolution Killilea, c'est le droit d'utiliser la langue minoritaire «en privé et en public » qui est évoqué.

8. Le gouvernement français a donné son approbation au texte de la Charte des droits fondamentaux de l'Union européenne lors de sa proclamation au sommet européen de Nice en décembre 2000 où les « minorités nationales » sont clairement évoquées dans le premier alinéa de l'article 21 ( « Est interdite, toute discrimination fondée notamment sur le sexe, la race, la couleur, les origines ethniques ou sociales, les caractéristiques génétiques, la langue, la religion ou les convictions, les opinions politiques, ou toute autre opinion, l'appartenance à une minorité nationale, la fortune, ... »), mais ce texte en est au stade d'une grande déclaration de principe, sans la force légale d'une convention telle que la CELROM, et la France ne reconnaît pas l'existence de «minorités nationales » sur son territoire. 
communes à une population donnée. La langue, il est vrai, est éminemment politique dans la mesure où, servant à communiquer et à identifier culturellement un groupe, elle peut être un instrument utile au pouvoir politique. De fait, la CELROM eut à connaître certaines difficultés avant d'être ouverte à la signature en 1992. Comme a pu l'expliquer le deuxième rapporteur de l'Assemblée parlementaire du projet de CELROM, Lluís Maria de Puig i Oliver (1995, p. 91), le premier projet de charte, élaboré entre 1984 et 1988 sous l'égide de la CPLRE, dut être dépouillé de certains aspects initialement proposés par les rédacteurs les plus impliqués. Il s'était agi principalement du droit à la « réparation historique ${ }^{9}$ et de l'inscription de la possibilité pour les langues régionales ou minoritaires d'être reconnues officielles. En outre, selon Lluís Maria de Puig i Oliver, « les mots "assurer", "garantir", furent en principe remplacés par "permettre”, "pouvoir", et certaines mesures normatives devinrent optionnelles... »10. Différents amendements furent ainsi apportés, en particulier le 11 septembre 1987, par la Commission qui adopta, moyennant cela, le texte. Le 21 octobre de cette même année, la CPLRE le renvoya à la Commission des affaires culturelles et sociales qui le remania encore. D'autres réunions eurent encore lieu au début de l'année 1988. Une version de la Charte, proposée par la CPLRE dans sa Résolution 192, lors de sa vingt-troisième session des 15-17 mars 1988, constitua un premier aboutissant notoire des travaux engagés dans cette perspective depuis 1984. L'Assemblée parlementaire accepta cette version dans son Avis 142 du 4 octobre 1988 sur la Résolution 192 (1988) sur les langues régionales ou minoritaires en Europe adoptée par la Conférence permanente des pouvoirs locaux et régionaux de l'Europe. Suivant les préconisations de cet Avis, le texte fut remis au Comité des ministres du Conseil de l'Europe en vue de son adoption comme convention. À ce stade, certains pays représentés dans le Comité, dont la France, refusèrent cette version de la Charte. Le Comité des ministres créa alors le Comité ad hoc d'experts sur les langues régionales ou minoritaires (CAHLR) qui dut revoir encore certains points du texte. Ce dernier fut encore amendé en vue de parvenir enfín à un accord du plus grand nombre possible d'États, tout en conservant l'objectif d'aboutir à une convention. Nous retiendrons deux modifications qui témoignèrent du poids prévisible des gouvernements dans cette discussion. La place des langues officielles d'État fut par exemple renforcée dans la version définitive du préambule. Ainsi, dans la version proposée par la Résolution 192, leur place était abordée de la façon suivante :

«Conscients du fait que la défense et le renforcement des langues régionales ou minoritaires dans les différents pays et région d'Europe, loin de constituer un obstacle aux langues nationales, représentent une contribution importante à la construction d'une Europe fondée sur les principes de la démocratie et de

9. Idée émise initialement par Henri Giordan dans le rapport qu'il remit au ministre de la Culture de l'époque, Jack Lang, Démocratie culturelle et droit à la différence. Paris : La Documentation française, 1982 , p. 47.

10. " els mots "assegurar", "garantir" sovint es van substituir per "permetre", "promoure" i algunes de les normes de compliment obligatori passaven a ser optatives... » (PUIG, 1995, p. 92). 
la diversité culturelle, dans le cadre de la souveraineté nationale et de l'intégrité territoriale ».

Ce sujet fut rendu plus explicite dans la version finale avec, au moins, une précision apportée dans la terminologie employée où « langue officielle » remplaça « langue nationale ». Cela permettait de préciser que le but de cette Charte n'était pas de proclamer des possibilités d'officialisation des langues régionales ou minoritaires, mais que d'autres langues pouvaient coexister dignement avec les langues officielles d'État. De ce point de vue, au-delà de l'affirmation des préćmincnces de statut en matic̀rc de droits linguistiques, l'expression « langue nationale » manquait de précision. La formulation destinée à rassurer, « loin de constituer un obstacle aux langues nationales » du quatrième paragraphe du préambule de la version de 1988 que nous venons de citer, fit, à la fin, l'objet, dans la version aboutie de la Charte, d'un paragraphe à part entière, formulé comme suit : « Soulignant la valeur de l'interculturel et du plurilinguisme et considérant que la protection et l'encouragement des langues régionales ou minoritaires ne devraient pas se faire au détriment des langues officielles et de la nécessité de les apprendre ». La connaissance, évidente pour tous, de ces langues officielles est présentée comme une nécessité, tandis que l'interculturel et le plurilinguisme sont présentés comme des valeurs, faisant peut-être écho au remplacement de « défense et renforcement » (des langues régionales...) par « protection et promotion »(des langues régionales...), les termes du texte de 1988 étant plus injonctifs que ceux de la CELROM de 1992. Ces modifications inscrivirent clairement dans le texte définitif que participait du socle de la Charte la distinction entre le caractère nécessaire des langues officielles d'État et celui, facultatif, des langues régionales ou minoritaires, entre la nécessité et la valeur. Également significatif nous paraît avoir été le changement intervenu dans la partie II de la Charte où l'article 5.1.a passa de :

«Les Parties s'engagent, en matière de langues régionales ou minoritaires parlées sur leur territoire, à prendre comme base de leur politique et dans leur législation et pratique les objectifs et principes suivants : a. la reconnaissance des langues régionales ou minoritaires en tant qu'attribut d'une communauté »

à, au début de l'article 7 de la version définitive de 1992 :

« En matière de langues régionales ou minoritaires, dans les territoires dans lesquels ces langues sont pratiquées et selon la situation de chaque langue, les Parties fondent leur politique, leur législation et leur pratique sur les objectifs et principes suivants : a. la reconnaissance des langues régionales ou minoritaires en tant qu'expression de la richesse culturelle ».

La modification importante porta ainsi sur l'alinéa a, tout en demeurant dans la logique de la Charte où, dès les premiers temps de son élaboration, il avait été précisé que seules les langues et non les minorités devaient faire l'objet de ce texte. Même si celui-ci comportait par endroits les mots « groupe » ou «minorité », cela n'aurait manifesté autre chose que le constat de la fonction première de communication que toute langue vivante est censée offrir. Ainsi qu'il avait 
déjà été clairement énoncé dans l'Exposé des motifs du Rapport portant avis sur la Résolution $192^{11}$, rédigé par Lluís Maria de Puig i Oliver, la référence à la « richesse culturelle » renvoyait là aussi à l'une des idées de fond de la Charte et il n'était donc pas illogique qu'elle figurât à la place de «communauté ». Le concept culturel de langue fut ainsi appuyé et consacré dans l'article 7 définitif (cf. article 5 dans la version de 1988), constitutif de toute la partie II de la Charte, qui intègre, avec le préambule et la partie I, l'ensemble des dispositions que les États s'engagent à appliquer en adhérant à la Charte ${ }^{12}$. La défense de la richesse culturelle de la langue permit de mettre l'accent sur le caractère effectif de mesures de protection et de promotion afin que celle-ci puisse accéder au plus grand nombre possible de fonctions sociales. Il en résulte que le point de départ est bien la langue et non le locuteur, et des indications détaillées seront données sur l'accès aux différents usages. Du point de vue des droits linguistiques, face à l'appréhension de certains États, particulièrement la France, cela permettait de ne pas aborder la question des droits individuels ou collectifs. Le Rapport explicatif est clair à ce sujet, dans ses paragraphes 11 (cf. infra) ou $17^{13}$. Pour autant, ces droits ne sont pas totalement absents puisque les langues sont bien émises par des locuteurs qui s'en servent pour communiquer entre eux. Or cette vérité première linguistique est ainsi traduite dans le Rapport explicatif de la Charte :

« La Charte vise à protéger et à promouvoir les langues régionales ou minoritaires, non les minorités linguistiques. Pour cette raison, l'accent est mis sur la dimension culturelle et l'emploi d'une langue régionale ou minoritaire dans tous les aspects de la vie des locuteurs. La Charte ne crée pas de droits individuels ou collectifs pour les locuteurs de langues régionales ou minoritaires. Néanmoins, les obligations des Parties en ce qui concerne le statut de ces langues et la législation interne qui devra être mise en place conformément à la Charte devront avoir un effet évident sur la situation des communautés intéressées et de leurs membre pris individuellement » (Rapport explicatif, 1993, § 11, pp. 5-6).

Si le risque d'une pression sociétale sur l'individu devait être prévenu et aménagé, le « groupe », la « communauté » ou telle autre entité désignée par quelque

11. "L'optique de la Charte est la défense de la diversité linguistique européenne en tant que dimension culturelle. Il ne s'agit pas des minorités, des ethnies ou des communautés, mais des langues si mal protégées que certaines sont en voie de disparition " (Exposé rédigé par L. M. de Puig i Oliver, Conseil de l'Europe, 14 septembre 1988, FDOC 5933, p. 1).

12. Sauf, toutefois, possibilité de réserves sur les paragraphes 2 à 5 de l'article 7 , conformément à l'article 21 de la Charte : «Tout État peut, au moment de la signature ou au moment du dépôt de son instrument de ratification, d'acceptation, d'approbation ou d'adhésion, formuler une ou plusieurs réserves aux paragraphes 2 a 5 de l'article 7 de la présente Charte. Aucune autre réserve n'est admise ».

13. "Le concept de langue tel qu'il est utilisé par la Charte s'articule essentiellement autour de la fonction culturelle de la langue. C'est la raison pour laquelle celle-ci n'est pas définie de manière subjective afin de consacrer un droit individuel, celui de parler "sa propre langue", la définition de cette langue appartenant à chaque individu ». 
terme que ce soit, mais dont le signifié est bien un ensemble de personnes qui se réfèrent à un même code linguistique pour communiquer entre elles ou qui, pour le moins, s'accordent à le valoriser dans cette fonction, ne peuvent être ignorés d'un point de vue sociolinguistique. Malgré les précautions et les engagements de la Charte à cet égard, tant dans le préambule qu'en tête de l'article 7 , la France s'opposa à ce qui, selon elle, pouvait faire allusion à des droits collectifs dans ce texte, en vertu des principes qu'elle défend traditionnellement. Outre que la Charte n'institue pas de droits individuels, mais une protection "à la carte" pour des langues peu répandues, la référence à certains endroits du texte à des groupes de locuteurs, essentiellement dans l'article 7, fut un des arguments qu'opposa ce pays. Au-delà, la France fit partie des pays, avec la Grèce, le Royaume-Uni et la Turquie, qui souhaitèrent que la Charte ne fût pas une convention mais une recommandation, par conséquent indicative et exemptée de l'obligation d'accomplissement. Ces quatre pays, pour des raisons diverses, exprimaient par là une crainte que cela ne renforce l'émergence d'entités culturelles, voire nationales, distinctes. La Grèce pouvait s'appuyer sur une tradition de protection ethnico-culturelle légitimée par une histoire de lutte contre la domination ottomane. La Turquie, niant l'existence et la pratique de toute différence culturelle socialisée autre que religieuse, pouvait s'abriter derrière une conception faussement égalitaire et extrêmement restrictive des droits culturels en général et linguistiques en particulier. Le Royaume-Uni, sans doute moins négatif par rapport aux droits linguistiques que ces deux autres pays, dans un contexte, à l'époque, de libéralisme autoritaire, développait une attitude méfiante à l'égard, notamment, du gaélique en Irlande du Nord, assimilé à la révolte catholique. L'État britannique était en outre clairement enclin, par tradition, à reconnaître des droits linguistiques aux individus plutôt qu'à des groupes minoritaires, d'où une crainte, à ce moment en tout cas, que la Charte ne contribue à favoriser l'émergence de ces communautés. La France rejoignait objectivement ces trois pays en réaffirmant son habituel rejet de tout ce qui pouvait, à ses yeux, contribuer à renforcer des liens communautaires dans le cadre d'un État-nation ne connaissant que des individus-citoyens. La tradition étatique dans ce pays de ne connaître que la seule langue française pour les usages officiels et publics était aussi en cause. En réalité, les mécanismes de contrôle prévus dans la version adoptée comme convention en 1992 sont réduits aux rapports publics régulièrement établis par les Parties au cours de la première année de mise en application de la Charte, puis tous les trois ans, et par un Comité d'experts nommé pour une période de six ans par le Comité des ministres. Aucun recours juridictionnel approprié n'est prévu en cas de non-respect des engagements. Il reste l'autocontrôle exercé par les États eux-mêmes et la force informative des rapports rédigés par des experts désignés ou proposés par les Parties ${ }^{14}$. Ces différentes garanties étaient destinées tant à préserver les prérogatives des États dans le dessein programmé de recevoir l'adhésion du plus grand nombre d'entre

14. Voir la partie IV de la Charte, avec l'article 15 sur les rapports périodiques, l'article 16 sur l'examen des rapports et l'article 17 sur le Comité d'experts. Voir également sur le sujet, CloteT I MiRO, 1994, pp. 557-562. 
eux qu'à s'assurer un minimum d'efficacité, ne serait-ce que grâce au pouvoir de l'information. Des oppositions demeurèrent malgré ce contrôle peu lourd $a$ priori et les amendements apportés au texte. Par exemple, en droit interne français, la production directe d'effet, en application de l'article 55 de la Constitution, serait en outre modulée au cas par cas, dans l'hypothèse d'une ratification, par la jurisprudence du Conseil d'État et de la Cour de cassation ayant à interpréter telle ou telle disposition de la Charte alléguée par des plaignants. Le Comité des ministres surmonta cependant ces réticences et conféra le rang prévu de convention à la Charte, faisant ainsi accéder, pour la première fois en droit international, la protection des droits linguistiques des langues peu répandues à un rang majeur.

Une des autres grandes caractéristiques de la Charte réside dans l'affirmation, dès le préambule, de l'imprescriptibilité du « droit de pratiquer une langue régionale ou minoritaire dans la vie privée ou publique ». La garantie d'accès de la langue à un usage public ne fut finalement pas démentie par des amendements qui auraient pu en réduire le principe. Parallèlement à cela, d'autres principes de base nous paraissent ressortir : le rejet dans la partie II de la Charte (article 7.2) de « toute distinction, exclusion, restriction ou préférence injustifiées portant sur la pratique d'une langue régionale ou minoritaire et ayant pour but de décourager ou de mettre en danger le maintien ou le développement de celle-ci ${ }^{15}$ et l'inscription du principe de discrimination positive ${ }^{16}$, pouvant correspondre en France à celui d'égalité par la différenciation ${ }^{17}$ - une aide active aux langues régionales ou minoritaires, une large place faite au caractère facultatif des

15. La version précédente de la Charte publiée à la suite de la Résolution 192 de la CPLRE précisait, dans son article 1.c, ce qui devait être entendu comme discrimination : "Par l'expression "discrimination", l'on vise toute distinction, exclusion, restriction ou préférence portant sur la pratique d'une langue ou l'appartenance à une minorité linguistique ayant pour but ou effet de décourager, de compromettre ou d'empêcher le maintien ou le développement d'une langue régionale ou minoritaire ou portant atteinte à l'égalité des droits des locuteurs de ces langues par rapport aux locuteurs des langues plus répandues dans les domaines de la vie privée ou publique ». Cela correspondait, tout en étant plus précis, à la citation que nous faisons de l'article 7.2. Il ressort de cette mise en parallèle que la formulation de l'article 7.2 se rapporte bien à l'idée de discrimination et que la référence à la notion de minorité linguistique a été entre-temps abandonnée, ce qui, nous l'avons vu, correspond aux choix de base du texte qui s'applique en droit aux langues et non aux minorités.

16. Cf. article 7.2 : «L'adoption de mesures spéciales en faveur des langues régionales ou minoritaires destinées à promouvoir une égalité entre les locuteurs de ces langues et le reste de la population ou visant à tenir compte de leurs situations particulières n'est pas considérée comme un acte de discrimination contre les locuteurs des langues plus répandues ».

17. Une des questions qui se posent au sujet des langues régionales en France est, à notre sens, celle des limites (contexte politique, évolution des mentalités, réalité et perception de la demande et de l'offre envisageable,...) de l'application à leur endroit du principe d'égalité substantielle comme complémentaire de celui d'égalité formelle. Le Principe d'égalité substantielle apparaît en France à partir de 1958, notamment dans la jurisprudence, à travers celui de l'égalité par la " différenciation » (cf. GRAU, 1992, pp. 99-100). Les lois spécifiques de 1982 et 1991 sur la Corse en furent par exemple des illustrations. Voir la Décision du Conseil constitutionnel 91290 DC du 9 mai 1991 à propos de la loi sur le statut de collectivité territoriale de Corse. 
mesures proposées, en particulier dans l'enseignement. Sur ce dernier point, les différentes dispositions détaillées dans la partie III, qui concernent l'enseignement, la justice, l'administration, les médias, les activités et équipements culturels, la vie économique et sociale et les échanges transfrontaliers, sont établies sur la base d'une protection minimale. Elles font des propositions facultatives ou modulées selon la réalité d'une demande et en fonction de la bonne marche des services concernés. En outre, aux termes de l'article 2, en dehors du préambule et de la partie II (article 7), qui s'applique « à l'ensemble des langues régionales ou minoritaires pratiquées sur son territoire et répondant aux définitions de l'article 1 », l'État qui ratifie « s'engage à appliquer un minimum de trente-cinq paragraphes ou alinéas choisis parmi les dispositions de la partie III de la présente Charte, dont au moins trois choisis dans chacun des articles 9, 10, 11 et 13 » (alinéa 2). Ces trente-cinq dispositions sont en outre censées être applicables, selon les termes de l'article 3, à un nombre déterminé de langues dont la liste est fournie par la Partie. De ce fait, des dispositions estimées trop contraignantes dans les domaines de la justice et des services administratifs dans des pays où elles n'auraient pas eu cours depuis parfois plusieurs siècles, comme en France pour l'occitan par exemple, peuvent être écartées dans le document de ratification, sinon à titre facultatif et en traduction du français, seule référence officielle. Ces dernières possibilités correspondent justement aux choix envisagés par la France en vue de son adhésion à la Charte en 1999 (cf. infra), les contraintes imposées par l'article 2 alinéa 2 de la CELROM étant les moins étendues dans les registres de communication les plus formels et liés au pouvoir tels que ceux qui découlent des articles 9 ("Justice") et 10 ("Autorités administratives et services publics").

La Charte fut finalement ouverte à la signature le 5 novembre 1992 et entra en vigueur le $1^{\text {er }}$ mars 1998 , le nombre des cinq ratifications nécessaires à cette fin ayant alors été atteint. En janvier 2002, sur les quarante-trois États membres du Conseil de l'Europe, douze avaient signé la Charte et seize l'avaient ratifiée. Parmi les quinze États également membres de l'Union européenne (UE), la France, l'Italie et le Luxembourg l'ont seulement signée, tandis que les huit pays qui l'ont ratifiée, l'Autriche, le Danemark, la Finlande, l'Allemagne, les PaysBas, l'Espagne, la Suède et le Royaume-Uni, parmi lesquels trois des plus importants de l'UE, représentent la moitié des membres du Conseil de l'Europe à avoir effectué cette démarche décisive (cf. supra).

Comme nous l'avons vu précédemment, le Parlement européen avait encouragé, dans la Résolution Kuijpers, le processus devant aboutir, au Conseil de l'Europe, à la CELROM. Il confirma cette intention dans la Résolution Killilea, adoptée le 9 février 1994, en incitant les gouvernements des États membres à y adhérer $^{18}$. Le Traité sur l'Union européenne, texte d'un autre poids politique,

18. « 6. soutient la Charte européenne des langues régionales ou minoritaires, à laquelle a été conférée la forme juridique d'une convention européenne, en tant qu'instrument à la fois efficace et souple de protection et de promotion des langues de moindre diffusion ; 7. invite les gouvernements des États membres qui ne l'ont pas encore fait à signer et leurs parlements à ratifier d'urgence cette 
signé à Maastricht le 7 février 1992 par les gouvernements des États membres de la Communauté européenne d'alors, dans son titre IX (article 128) "Culture", confirme sa coopération dans ce domaine « en particulier avec le Conseil de l'Europe », mais ne contient qu'une formule générale au sujet des cultures régionales ${ }^{19}$. La Charte des droits fondamentaux de l'Union européenne est proclamée lors du sommet de Nice par le Conseil des ministres, la Commission et le Parlement de l'Union européenne. En comparaison, la Convention-cadre pour la protection des minorités nationales, également en vigueur depuis le début de l'année 1998 et contenant un certain nombre de dispositions sur les langues minoritaires (cf. supra), a été ratifiée à ce jour par trente-quatre États ${ }^{20}$. Les questions concernant spécifiquement les langues peu répandues restent peut-être plus délicates à traiter et la CELROM est, dans ce domaine, un texte à la fois détaillé et élaboré où les questions d'aménagement linguistique sont abordées de façon approfondie. Il apparaît néanmoins que le rythme de croissance des signatures et ratifications, après avoir été relativement lent, est plus rapide depuis le début de l'année 2000.

\section{DE LA LANGUE ET DE SON TERRITOIRE}

À côté des questions qui tiennent aux principes et au contenu de la Charte, il en est d'autres, également importantes, qui découlent de l'intitulé même de ce texte. Ainsi en va-t-il des notions de langue régionale et de langue minoritaire que nous allons à présent aborder en relation, particulièrement, avec la notion de territoire. La désignation elle-même de « langue régionale » se réfère directement à l'idée de territoire.

\subsection{LANGUES RÉGIONALES OU MINORITAIRES ET TERRITOIRE}

L'expression « langue régionale » est répandue en France et pourrait maintenant avoir tendance à être de plus en plus utilisée en Europe du fait de la CELROM. L'histoire de cette notion, passée dans l'usage courant depuis ces dernières décennies, au moins en France, témoigne de débats et de représentations qui illustrent les conceptions apparues à l'égard de ces langues « régionales ». En Aquitaine, par exemple, l'aire de la langue basque se prolonge de l'autre côté de la frontière, en Espagne, où elle est caractérisée par deux autres appellations

convention, en choisissant à tout moment d'appliquer les paragraphes qui répondent le mieux aux besoins et aux aspirations des communautés linguistiques concernées » (Résolution (Killilea) sur les minorités linguistiques et culturelles dans la Communauté européenne, adoptée le 09/02/1994 par le Parlement européen, PE 179.621).

19. « La Communauté contribue à l'épanouissement des cultures des États membres dans le respect de leur diversité nationale et régionale, tout en mettant en évidence l'héritage culturel commun $»$ (article 128.1).

20. Seuls trois pays ne l'ont pas encore signée : la Belgique, la France et la Turquie. La France ne reconnaît pas la notion de minorité. 
légales, celle de « langue propre » ${ }^{21}$ et celle de «langue officielle », co-officielle en réalité avec le castillan. Dans le Val d'Aran, également en Espagne, l'occitan lui-même est également langue propre, depuis 1983, et co-officielle, avec le castillan et le catalan, depuis $1990^{22}$. Du côté français, les deux langues, basque et occitane, sont, officiellement, des langues régionales.

En France, l'expression « langue régionale » commença à apparaître clairement à partir du milieu des années soixante. La circulaire ministérielle $\mathrm{n}^{\circ} 66-$ 361 du 24 octobre 1966 prévoyait la mise en place des Commissions académiques d'ćtudes régionales et se référait aux langues régionales parlées en France. La régionalisation de la France était en débat et commençait à déboucher sur les premiers éléments de réponse institutionnelle comme en témoignèrent les décrets des 7 janvier 1959 et 2 juin 1960 fixant les circonscriptions d'action régionale et du 14 mars 1964 instituant les préfets de région. Ces langues régionales furent celles que la loi Deixonne du 11 janvier 1951 sur l'enseignement des langues et dialectes locaux avait énumérées - sans les qualifier encore de « régionales »- plus quelques autres, comme le corse par exemple, qui, à ce titre, furent reconnues une vingtaine d'années plus tard. Les langues concernées, que l'unicité respective de chacune implique ou non l'identification de variantes dialectales, supposent implicitement une implantation territoriale traditionnelle ayant clairement marqué la culture d'une portion de l'ensemble national. On ne saurait isoler cette approche institutionnelle, qui aura ses effets dans le langage courant lorsque l'on parle de langues régionales en France, d'autres qui proviennent d'autres lieux d'élaboration conceptuelle. À ce propos, nous rappellerons ici celle qui se détache du texte de la CELROM. Cette convention donne pour égales en droit, dès son intitulé, à travers l'emploi de la conjonction « ou », les désignations « langue régionale » et « langue minoritaire ». Plus précisément, le Rapport explicatif indique, dans son paragraphe 18, que « L'adjectif 'régional' concerne les langues parlées dans une partie limitée du territoire d'un État dans laquelle elles peuvent, par ailleurs, être parlées par la majorité des citoyens » (ibid., p. 7). Or si une langue régionale peut y être parlée majoritairement, cela n'est pas non plus forcément le cas. L'adjectif « minoritaire », selon ce même rapport, se réfère uniquement à l'aspect quantitatif des locuteurs qui ne sont pas regroupés «sur une partie déterminée d'un État » ou bien qui se trouvent en nombre inférieur, dans une région déterminée, par rapport aux autres personnes qui parlent « la langue majoritaire de l'État » 23.

21. Voir, entre autres études sur ce sujet, Solé I Durany, 1996 ; Ferret I JaCAS, 1999 ; Mirambell i ABANCó, 1999.

22. Voir l'article 2, alinéa 1 de la loi sur le régime spécial du Val d'Aran (13 juillet 1990) : Er aranés, varietat dera lengua occitana e pròpia d'Aran, ei oficiau ena Val d'Aran. Tanben ne son eth catalan $e$ eth castelhan, d'acòrd damb er article 3 der Estatut d'Autonomia de Catalonha (L'aranais, variété de la langue occitane et propre du Val d'Aran, y est officiel. Le catalan et le castillan le sont également, en accord avec l'article $3 \mathrm{du}$ Statut d'autonomie de la Catalogne).

23. "L'adjectif "Ininoritaire" se réfere aux situations où soit la langue est parlée par des personnes qui ne sont pas concentrées sur une partie déterminée d'un État, soit elle est parlée par un groupe de personnes qui, bien que concentré sur une partie du territoire d'un État, est numériquement inférieur à la population dans cette région qui parle la langue majoritaire de l'État » (Rapport explicatif, 1993). 
Il en résulte que « minoritaire » pourra être utilement associé à « régionale » pour une langue qui, au fil du temps, n'aurait fini par ne concerner qu'un nombre réduit de locuteurs à l'intérieur de son territoire d'origine. Il est précisé, dans l'article 1 de la Charte, que l'expression « langues régionales ou minoritaires » concerne les langues « pratiquées traditionnellement sur un territoire d'un État par des ressortissants de cet Etat qui constituent un groupe numériquement inférieur au reste de la population de l'État et différentes de la (des) langue(s) officielle(s) de cet État ». Les dialectes de la (des) langue(s) officielle(s) ne sont pas concernés. Le Rapport explicatif de la Charte interprète l'expression « langues pratiquées traditionnellement » comme devant s'appliquer à des «langues historiques, c'est-à-dire qui sont parlées depuis une longue période dans l'État en question » (ibid., $\S 31$, p. 11). Quant au fait de savoir ce que l'on entend par « langue », les dialectes d'oil en France seraient ainsi exclus du champ d'application de la Charte si, après avoir été identifiés comme des langues par leurs promoteurs, ils n'avaient finalement été de plus en plus admis à ce titre par des instances propres à leur conférer un surcroît de légitimité ${ }^{24}$. Outre ce qui est explicitement dit dans l'article 1 de la Charte au sujet des dialectes de la, ou des, langue(s) officielle(s) de l'État, le Rapport explicatif précise que « la charte ne concerne pas les variations locales ou les différents dialectes d'une même langue » (ibid., § 32, p. 11).

Le risque existait de favoriser l'émergence de langues s'autonomisant en tant que telles alors qu'elles avaient jusque-là été décrites et perçues comme des dialectes primaires ${ }^{25}$. Il peut être estimé qu'il revient aux promoteurs et linguistes impliqués d'assumer les choix d'une normalisation linguistique « monostandardisatrice » tendant à privilégier d'abord une variété standard, ou qui module la modernisation de la langue entre standardisation et variation, lorsque cette dernière est restée vivante et fonctionnelle. Un choix peut aussi être fait d'aménager une place à la variation traditionnelle de la langue et d'en intégrer les raisons d'être dans un schéma d'ensemble de revitalisation linguistique. Les concepts de «polyélaboration », de «polynomie » et de « standardisation polycentrique », par exemple ${ }^{26}$, illustrent le pôle le plus enclin à intégrer la variation linguistique

24. Certaines langues d'oïl, comme le picard, le gallo et le poitevin-saintongeais, principalement, font l'objet de cours d'initiation ou en vue de la préparation d'une épreuve facultative au baccalauréat. De plus, le Rapport Cerquiglini, même s'il a été sans effet puisque la Charte n’a pas été ratifiée par la France cette fois-ci, a pu contribuer à leur légitimité : «Par définition, les variétés de la langue officielle ne sont pas du ressort d'un texte qui entend protéger les langues minoritaires, rendues précaires par l'extension, le rayonnement et l'officialisation de cette langue. Il convient donc de préciser la situation dialectale du français "national et standard". Que l'on adopte, pour expliquer sa genèse, la thèse traditionnelle et contestable d'un dialecte d'oïl (le supposé francien) "qui aurait réussi" aux dépens des autres, ou que l'on y voie la constitution très ancienne d'une langue commune d'oïl transdialectale, d'abord écrite, puis diffusée, le français "national et standard" d'aujourd'hui possède une individualité forte, qu'a renforcée l'action des écrivains, de l'État, de l'école, des médias » (CERQuiglini, 1999b).

25. Cf., pour cette terminologie, Coseriu, 1988.

26. Cf., pour la « polyélaboration », MULJACIC, 1986, p. 57 ; pour la « polynomic », concept développé à partir du cas du corse, MARCELLESI, 1988, p. 809 ; et pour la « standardisation polycentrique », STEWART, 1968, pp. 534-535. 
dans ses approches de la standardisation. La notion de « modalité linguistique », souple et large, qui apparaît dans l'article 3 alinéa 3 de la Constitution espagnole de $1978^{27}$, a permis de répondre à des attentes concernant tant des langues parfaitement identifiées dans leur indépendance comme, entre autres, le basque ou le catalan que des variantes du continuum castillan telles que le castillan andalou ou « modalité andalouse du castillan ». Ces questions portent en ellesmêmes celle de la protection, voire de la promotion, du dialecte, avec le risque - ou le pari - que ce dernier finisse par être érigé en langue autonome à la faveur de mesures valorisantes. Cela peut favoriser certaines formes de complémentarité comme simplement intégrer la réalité sociolinguistique de langues dont la transmission intergénérationnelle s'effectue à travers les formes héritées, dialectales $^{28}$. Par ailleurs, le fait de ne pas intégrer une protection des dialectes pouvait aussi inciter à les reconnaître comme langues afin de les faire bénéficier de mesures découlant d'une ratification de la CELROM. Toutefois, la présence d'une formule adéquate, intégrant une approche de la variation diatopique de chaque langue, minoritaire ou pas, était peut-être envisageable, laissant ainsi aux « dialectes » évoqués par la Charte une autre issue que celle de la non-prise en compte ou de l'accès au statut de langue. Peut-être dans le souci d'éviter, en reconnaissant les « dialectes », qu'ils ne contribuent à affaiblir des langues déjà peu répandues ou spécialement minorées, ou des langues d'État que leurs défenseurs institutionnels auraient pu croire ainsi menacées, le risque a été pris par les rédacteurs de la CELROM de favoriser indirectement la promotion au rang de « langues » de certains dialectes historiques. On pourrait estimer, il est vrai, que cela a pu contribuer à en confirmer l'identité ou l'émergence. Ce sujet pouvant se révéler un peu complexe, déjà à l'intérieur des États concernés, la détermination de ce qui entre dans la catégorie «langue » fut ainsi laissée à leur appréciation à travers la liste des langues devant faire l'objet de la partie III de la Charte qu'ils doivent fournir au moment de la ratification.

On comprendra mieux que, dans le rapport que remit le linguiste Bernard Cerquiglini au Ministre de l'éducation nationale et au Ministre de la culture en 1999 (Cerquigligni, 1999b) pour contribuer à l'étude de l'adhésion de la France à la Charte, étaient identifiés comme « langues d'oïl » ${ }^{29}$ des dialectes primaires

27. La riqueza de las distintas modalidades lingüísticas de España es un patrimonio cultural que será objeto de un especial respeto y protección (La richesse des différentes modalités linguistiques de l'Espagne constitue un patrimoine culturel qui fera l'objet d'un respect particulier et de protection).

28. À ce sujet, la Recommandation $n^{\circ} 928$ de l'Assemblée parlementaire du Conseil de l'Europe avait évoqué en 1981 l'emploi du dialecte dans l'enseignement préscolaire : « Au niveau humain, l'adoption progressive de la langue maternelle dans l'éducation des enfants (emploi du dialecte au niveau oral dans le préscolaire, et des formes normalisées de la langue maternelle dans l'enseignement primaire pendant lequel sera introduite ensuite peu à peu, à côté de la langue maternelle, la langue majoritaire du pays) » (article 4.b).

29. Les explications fournies l'année précédente par Bernard Poignant dans son rapport (Langues et cultures régionales. Rapport de Monsieur Bernard Poignant, Maire de Quimper, à Monsieur Lionel Jospin Premier Ministre. Juillet 1998, 72 p.) sont moins précises à l'égard de ces langues : « formes régionales du français » (p. 8), « Les langues d'oïl sont devenues des français régionaux » (p. 9). 
du français. Pour cela, il estima devoir prendre acte de l'accroissement, au fil du temps, de la distance linguistique entre le standard et ces dialectes premiers et, implicitement, de l'action de mouvements culturels qui ont œuvré dans le dessein de les faire identifier comme tels. Il en vint en même temps à proposer que l'on considère comme dialectes du français des variétés régionales tertiaires, les «français régionaux » ${ }^{30}$, pour reprendre la terminologie de Coseriu (1988).

Le « territoire », au sens de la Charte, appelle d'autres précisions. Il convient, ici, de citer le Rapport explicatif (1993, § 33, pp. 11-12) à ce sujet :

« Il ne s'agit pas seulement du territoire dans lequel cette langue présente un caractère dominant ou majoritaire, car beaucoup de langues sont devenues minoritaires même dans les zones constituant leur assise territoriale traditionnelle. Si la Charte prend principalement en compte des langues qui comportent une assise territoriale, c'est parce que la plupart des mesures qu'elle préconise nécessitent la définition d'un champ géographique d'application autre que celui de l'État dans son entier. Bien entendu, des situations existent où plus d'une langue régionale ou minoritaire est parlée dans un territoire déterminé ; la Charte couvre également ces situations ».

Des langues dites régionales sont souvent devenues également minoritaires dans leur territoire d'implantation traditionnel au cours des dernières décennies. Cela est la règle en France par exemple, mais relève aussi d'une tendance plus largement établie. Il paraît ainsi difficile de dissocier les deux notions, « régionale » et «minoritaire », mais un tel contexte n'est pas non plus représentatif de tous les cas en Europe. En effet, des langues minoritaires ne le sont que par rapport à la langue de l'État mais pas forcément à l'intérieur de la zone où elles sont implantées. Des langues régionales qui ne sont pas minoritaires dans leur aire traditionnelle existent en plusieurs endroits, dans des situations de diglossie ${ }^{31}$ et de bilinguisme avec des langues officielles d'État.

30. «Il en résulte que l'on tiendra pour seuls "dialectes" au sens de la Charte, et donc exclus, les "français régionaux", c'est-à-dire l'infinie variété des façons de parler cette langue (prononciation, vocabulaire, etc.) en chaque point du territoire. II en découle également que l'écart n'a cessé de se creuser entre le français et les variétés de la langue d'oïl, que l'on ne saurait considérer aujourd'hui comme des "dialectes du français" ; franc-comtois, wallon, picard, normand, gallo, poitevin-saintongeais, bourguignon-morvandiau, lorrain doivent être retenus parmi les langues régionales de la France ; on les qualifiera dès lors de "langues d'oïl", en les rangeant dans la liste " (CERQuiglinI, 1999b).

31. La notion de diglossie est entendue ici dans le sens élargi de situation de contact hiérarchisé entre des variétés linguistiques distinctes (cf. FishmaN, 1967) pouvant être des variétés d'une même langue (sens initial développé par Charles A. Ferguson) ou des langues différentes, ce dernier cas étant plutôt celui qui correspond aux situations abordées par la Charte. Ce contact asymétrique, audelà de cette approche, a également pu être décrit depuis par des sociolinguistiques impliqués comme un processus par essence dynamique et porteur de tensions latentes ou ouvertes (apport du Groupe de sociolinguistique catalane, notamment, à partir de la fin des années soixante). Ainsi, la notion de conflit linguistique a-t-elle pu être avancée pour relayer celle de diglossie, employée au départ pour désigner des situations relativement stables. Il est non moins vrai que certaines situations sont plutôt stables, et se prêtent aux premières interprétations, et d'autres, plus conflictuelles, correspondent mieux aux suivantes. 
Il se détache aussi de cette explication que le territoire de référence pour la Charte est autant le territoire linguistique, c'est-à-dire celui des locuteurs, que le territoire d'intervention administrative, tel qu'il peut être déterminé dans le cadre de la mise en application de mesures planifiées ayant pour but de protéger une langue régionale utilisée et/ou faisant l'objet de demandes dans ce sens. De ce fait, le nombre de locuteurs n'est pas retenu par la Charte comme un élément premier pour l'identification du territoire linguistique. Par ailleurs, quant à l'implantation territoriale des langues, comme dans la tradition française, la Charte exclut a priori les langues des migrants du champ d'application de la notion de langue régionale ou minoritaire. Au-delà, les « langues dépourvues de territoire » sont en principe traitées à part, tant la référence à une implantation territoriale constitue un des fondements, à portée pratique et administrative, de la protection linguistique pour cette convention. Ces langues sans implantation territoriale peuvent quand même répondre à la définition de l'article 1, étant, de fait, elles aussi, « pratiquées traditionnellement » sur le territoire de l'État. Le Rapport explicatif $(1993, \S 36$, p. 12) mentionne ainsi le yiddish et le romani comme exemples de langues qui répondent à cette dernière configuration. Des restrictions sont néanmoins évoquées pour ces langues dans l'article 7 alinéa 5. « À défaut d'assise territoriale, seule une partie limitée de la Charte est susceptible de s'appliquer à ces langues », est-il précisé en tête du paragraphe 37 du Rapport explicatif.

Le territoire linguistique, perçu à travers une acception neutre d'espace intégré ou délimité par une gestion administrative, n'en demeure pas moins susceptible d'être sujet à des évaluations mettant en évidence une attitude de subordination dans un sens ou de repliement dans un autre. Ainsi, l'expression « langue régionale » a-t-elle pu faire l'objet d'interprétations critiques tandis que la Charte contribue à en valider l'emploi.

\subsection{LIMITES DE LA DÉSIGNATION «LANGUE RÉGIONALE 》}

L'expression « langue régionale » s'est affirmée au cours de la deuxième moitié $\mathrm{du} \mathrm{XX}^{\mathrm{e}}$ siècle comme une désignation permettant d'identifier certaines langues dont l'avenir était menacé par des faits de civilisation et/ou d'unitarisme linguistique. Un mouvement de prise de conscience international s'est développé en leur faveur. Des effets s'en sont fait sentir en France comme ailleurs et il est admis maintenant qu'il convient de les protéger, voire de les promouvoir, afin de contribuer à « maintenir et développer les traditions et la richesse culturelle de l'Europe », ainsi qu'il est dit dans le préambule de la CELROM. Dans un souci de stabilité, de maintien des unités stato-nationales, mais aussi avec la conviction de l'intérêt pragmatique et culturel de préserver les acquis des langues de plus grande diffusion en faveur d'une communication aussi élargie que possible, il est également admis, comme c'est aussi le cas dans ce même préambule, que cette protection ne saurait nuire aux langues officielles d'État ${ }^{32}$.

32. «Soulignant la valeur de l'interculturel et du plurilinguisme et considérant que la protection et l'encouragement des langues régionales ou minoritaires ne devraient pas se faire au détriment des langues officielles et de la nécessité de les apprendre » (Préambule de la CELROM). 
À l'échelle de l'Union européenne, le Parlement européen s'est de plus en plus aligné, au cours de ces dernières années, sur la terminologie développée par le Conseil de l'Europe, en utilisant l'expression « langue régionale ou minoritaire », ne serait-ce qu'à travers les résolutions visant à soutenir cette organisation dans son entreprise pour faire aboutir la Charte en question. Aussi bien, certains autres textes qui émanent du Parlement européen ont utilisé d'autres fois les expressions «langues et cultures minoritaires » ${ }^{33}$, « langues et cultures des minorités régionales et ethniques » ${ }^{34}$, « minorités linguistiques et culturelles » ${ }^{35}$. Par ailleurs, le Bureau européen pour les langues moins répandues, soutenu, depuis sa création en 1982, par la Commission européenne, emploie dès son intitulé une autre formulation censée être plus neutre. En réalité, la Charte utilise cette formulation dans son article 3 alinéa 2 au sujet des modalités d'application, en l'affectant à un type de langue officielle : « (...) qu'elle appliquera (...) à d'autres langues régionales ou minoritaires, ou à d'autres langues officielles moins répandues ». Il est cependant évident que cet emploi isolé doit être mis en relation avec l'article 1 des « définitions » où il apparaît clairement que si les langues régionales ou minoritaires incluent les langues « différentes de la (des) langue(s) officielle(s) » (alinéa a. ii) des États, elles désignent aussi bien des langues sans statut légal très déterminé que d'autres, déjà officielles, mais seulement à des échelons régionaux, infra-étatiques. Elles y sont d'ailleurs le plus souvent coofficielles ${ }^{36}$, en association avec une langue officielle d'État à laquelle correspond un régime de personnalité à l'échelle du pays.

Dans d'autres textes à portée internationale, publiés ou actuellement à l'étude en vue de déboucher sur des réglementations supranationales, le non-emploi de la notion de langue régionale a, au contraire, été voulu. L'idée de protection des groupes linguistiques minoritaires a pu lui être préférée, comme c'est le cas dans le Rapport Euromosaic (1996), rédigé et publié à la demande de la Commission européenne en 1996. Ce rapport met en effet l'accent non pas sur la langue en tant que telle, comme le fait la CELROM, mais sur le contexte social et économique dans lequel évolue une langue :

«Sous bien des aspects, l'équipe a senti qu'elle devait s'écarter de la normativité de l'orthodoxie sociolinguistique et de la sociologie du langage. Cette normativité se traduit par une forte tendance à la réification de la langue

33. Résolution sur les mesures en faveur des langues et des cultures minoritaires (J.O. C 68 du 14/03/1983, p. 103).

34. Résolution (Kuijpers) sur les langues et cultures des minorités régionales et ethniques de la Communauté européenne, op. cit.

35. Résolution (Killilea) sur les minorités linguistiques et culturelles dans la Communauté européenne, op. cit.

36. Jusqu'à présent, le cas présente une exception avec l'italien, « langue officielle moins répandue ", officielle dans le canton du Tessin et coofficielle au niveau du gouvernement confédéral, aux côtés de l'allemand, du français et du romanche. Par ailleurs, à l'intérieur du canton des Grisons, le romanche et l'italien sont en fait coofficiels, à côté de l'allemand, pour un certain nombre de services administratifs importants, l'enseignement, la justice. 
aboutissant à des concepts tels que le "changement linguistique" ou le "contact linguistique", lesquels extérieurement sont dépourvus de tout contexte social ou économique »(Euromosaic, 1996, p. II).

Il y est question de «groupes linguistiques ». La CELROM et le Rapport Euromosaic n'ont cependant pas la même fonction première. La Charte, destinée à produire du droit dans ce domaine, à l'intérieur des États membres du Conseil de l'Europe, ne pouvait être établie que sur un socle propre à recevoir la plus grande adhésion possible. L'évocation du droit des groupes est perçue par certains États - la France, en particulier - comme contradictoire avec leur conception de l'unité de la nation. La structure fédérale, ou « des autonomies » comme en Espagne, autorise davantage la prise en compte légale de la socialisation des usages linguistiques. La considération de cet aspect est, en revanche, à peu près incontournable d'un point de vue sociolinguistique et un aménagement linguistique ne peut ignorer la réalité de la communauté linguistique, même s'il est convenu d'affirmer la langue avant le groupe auquel elle est liée. André Martinet rappela en son temps cette vérité première selon laquelle, « dès qu'une langue est conçue comme un instrument de communication s'adaptant aux besoins du groupe qui l'utilise : "communication" implique "communauté" » (Martinet, 1969, p. 151) ${ }^{37}$. Le débat porte aussi sur ce qui sera entendu par les « besoins du groupe », sachant que ceux-ci peuvent relever d'une fonctionnalité effective au moment où se pose cette question, ou bien faire l'objet d'un investissement identitaire de la part de la communauté qui se sent impliquée, en vue de proposer la mise en place de conditions minimales propres à susciter le maintien et/ou l'apparition, ou la réapparition, desdits besoins ${ }^{38}$. En fait, cette question en appelle d'autres, importantes quant au caractère effectif de la mise en place de ces conditions et quant à la masse critique utile et nécessaire au résultat qui en est attendu. Enfin, la quête d'identité culturelle en tant que telle doit-elle être comptabilisée parmi ces besoins ou non ? ${ }^{39}$. La CELROM ne

37. Les quatre nuances qu'il apporta à cette assertion ne sont pas moins pertinentes. Nous en retiendrons ici les premiers énoncés respectifs qui suivent : "Aucune communauté n'est linguistiquement homogène " (variations d'un individu à l'autre pour un code commun), « De nombreux locuteurs appartiennent à deux ou à plus de deux communautés » (régions bilingues ou variété topolectale + standard), « De nombreuses personnes utilisent concurremment différents styles de la même langue », « De nombreuses personnes qui n'utilisent jamais qu'un seul style ou une seule langue comprennent, par contre, différents styles ou différentes langues » (connaissance passive) (MARTINET, 1969, pp. 151-152).

38. Cela nous renvoie aux notions de « cercle fonctionnel » ainsi que de «fonctions sociales de la langue " (output) et de "fonctions linguistiques de la société » (input), développées par Lluís V. Aracil en relation avec son interprétation de la normalisation linguistique. Cette dernière apparaît comme le résultat d'un feedback négatif ayant provoqué un effort compensatoire propre à susciter le retour ou l'apparition de fonctions sociales efficientes pour la langue concernée (ARACIL, 1982, pp. 23-38).

39. Louis-Jean Calvet n'apporte pas de réponse explicite à cette question. Toutefois, reprenant de façon critique l'approche de type cybernétique de Lluís V. Aracil (cf. supra), il doute que l'on puisse faire émerger efficacement de nouvelles fonctions sociales pour une langue peu répandue à l'aide de besoins linguistiques nouveaux si cette dernière ne s'avère plus fonctionnelle ou ne fait plus suffisamment l'objet de demandes sociales spontanées. Selon cette approche, de nouvelles demandes 
répond pas à ces dernières interrogations mais n'en ignore pas le contexte puisque les mesures d'aménagement linguistique qu'elle suggère sont destinées à favoriser l'émergence de conditions favorables à une revitalisation des langues régionales ou minoritaires en Europe, son objectif premier, par choix politique et méthodologique, demeurant celui de protéger les langues régionales ou minoritaires en tant que telles (Rapport explicatif, 1993, $\$ 17$, p. 7). Dans tous les cas, si la langue possède encore une existence effective, cela ne se peut qu'avec des locuteurs qui l'utilisent d'une façon ou d'une autre pour communiquer entre eux. Dans une vision ouverte, cela peut aboutir à faire en sorte que le groupe ne se referme pas exagérément sur lui-même et à proposer la langue, régionale ou pas, comme un bien de la nation dans son ensemble, même si un principe de réalité fait admettre que c'est plutôt telle partie de la nation et du territoire de l'État qui est d'abord concernée. Dans une vision étroite, cela peut déboucher sur une hiérarchisation renforcée des contacts de langues, le particulier pouvant toujours être suspecté de contribuer à diviser le général, premier garant des conditions de l'égalité et de la liberté des citoyens.

La Déclaration universelle des droits linguistiques, officiellement engagée depuis 1996 sur une voie devant la conduire à terme à devenir une convention internationale des Nations Unies, rejette l'appellation « langue régionale » pour le caractère ambigu qu'elle présenterait. Il y est même estimé qu'elle a pu être utilisée pour « restreindre les droits d'une communauté linguistique »:

«La présente déclaration part du principe que les droits de toutes les communautés linguistiques sont égaux et indépendants du statut juridique ou politique de leur langue en tant que langue officielle, régionale ou minoritaire ; les expressions "langue régionale" et "langue minoritaire" ne sont pas utilisées dans la présente Déclaration car il y est fréquemment recouru pour restreindre les droits d'une communauté linguistique, même si la reconnaissance d'une langue comme langue minoritaire ou régionale peut parfois faciliter l'exercice de certains droits » (article 5).

En France même, l'expression « langue régionale » est sujette à débat. Déjà, dans son Rapport au Ministre de la culture, publié en 1982, Démocratie culturelle et droit à la différence, Henri Giordan pensait qu'elle était inadéquate car ces langues ne coïncident pas avec les régions administratives françaises. En fait, cette situation constitue la règle générale à laquelle ne dérogent que de rares exceptions. En outre, ces langues concernent des aires qui peuvent déborder des frontières. Elles peuvent aussi être utilisées loin du territoire où elles sont traditionnellement implantées, dans des agglomérations en particulier (Giordan, 1982, pp. 50-51). Cet auteur préconisa la désignation « langue minoritaire », plus neutre à son sens car présentant, entre autres arguments, celui d'être plus répandue internationalement et de pouvoir convenir aussi bien à des langues

sociales peuvent être le fait de militants de là langue et leur caratère artificiel au départ ne débouche pas forcément sur une réorganisation effective des fonctions linguistiques de la société si la langue n'est plus assez employée et valorisée (CALVET, 1993, pp. 177-199). 
implantées dans une région qu'à d'autres qui ne le sont pas (ibid., p. 52). Les défenseurs des langues concernées regrettent généralement le sens lié à une situation subalterne dont serait porteuse l'expression « langue régionale ». Localement, le paradigme découlant de la notion de région fait par exemple l'objet d'une réflexion critique au sein du mouvement occitan de nos jours. Nombre de ses représentants les plus connus se déclarent anti-régionalistes et « antiracines », par refus, à la fois, de l'idée de subordination contenue dans l'adjectif « régional » et de celle d'enfermement dans un particularisme ${ }^{40}$. En ce sens, ils se reconnaissent dans le cadre français dans la mesure où ce dernier conjuguerait la tradition d'universalisme - qu'ils ont intégrée - et sa pluriculturalité originelle. Cela implique à leurs yeux de repenser un projet français dans lequel l'universalisme tendrait vers autre chose qu'une abstraction réductrice et monolithique.

Il se dégage en commun de l'exemple français, comme des références, notamment, à la CELROM, que la notion de langue régionale, associée ou non à celle de langue minoritaire, concerne des langues menacées de disparaître à court ou plus long terme. La langue régionale n'y apparaît pas, dans le discours officiel, comme une « langue propre » potentielle, susceptible, de ce seul fait, de justifier de droits étendus pouvant aller jusqu'au caractère officiel, ainsi que cela se dégage de la législation espagnole en vigueur ou, plus diffusément, de la Déclaration universelle des droits linguistiques (cf. supra). En attendant, c'est par conséquent cette désignation de «langue régionale » qui continue à être majoritairement et officiellement utilisée en France. Elle est consacrée par la CELROM. Or un des sujets importants sur lesquels différents débats et documents ont vu le jour en relation avec cette "question" a justement été celui de la notion de territoire linguistique dont nous venons de voir qu'elle est liée à celle de langue régionale. Malgré cette consécration apportée par la Charte à la « langue régionale », mais aussi, pour d'autres raisons précédemment évoquées, la France a, pour le moment, refusé de ratifier cette convention. Le débat qui entoura la question de l'adhésion de la France à la Charte (voir, à ce sujet, Renaut, 2000) vit surgir des interrogations de fond au sujet de la notion de langue régionale. Elles ne provinrent pas tant du milieu militant que de spécialistes choisis par le pouvoir pour préparer la prise de décision. Ces questions renvoyèrent en fait aux conceptions de la nation, du territoire national et de la territorialité linguistique. Des points de vue critiques furent en particulier émis dans les rapports Carcassonne et Cerquiglini, rédigés en vue de préparer la signature puis la ratification de la Charte par la France. Dans le rapport que remit le juriste Guy Carcassonne au Premier ministre en 1999, apparaît une distinction entre « langue régionale » et « langue minoritaire », ces dernières pou-

40. Ce discours est porté en particulier par des écrivains et artistes tels que Félix Castan ( $\dagger$ ), Bernard Lubat, Bernard Manciet, Claude Sicre et d'autres. 
vant répondre aux indications contenues dans le paragraphe 36 du Rapport explicatif de la Charte ${ }^{41}$ :

«- en considérant comme langue dite régionale toute langue historiquement et géographiquement parlée par des Français sur le territoire national, métropole comme outre-mer, sans que, à ce stade, il y ait lieu à attacher une importance excessive au nombre des locuteurs actuels ;

- en considérant comme langue minoritaire toute langue parlée par un nombre significatif de Français, à condition qu'elle ne soit pas langue officielle dans un pays étranger, et puisse être menacée de disparaître » (Carcassonne, 1998, p. 129).

Jouant aussi sur l'emploi de l'adjectif « historique » dans le préambule («protection des langues régionales ou minoritaires historiques de l'Europe ») et de l'adverbe «traditionnellement » dans l'article 1.a.i (« les langues pratiquées traditionnellement... ») de la Charte, Bernard Cerquiglini reprit cette suggestion dans son Rapport ${ }^{42}$ en incluant, parmi les soixante-quinze langues à protéger, le berbère, l'arabe dialectal, le yiddish, le romani chib et l'arménien occidental. L'argumentation qu'il développa répondait à la définition donnée par Guy Carcassonne de la « langue minoritaire ». Il retint que des langues parlées (berbère, arabe dialectal) dans d'anciens départements français d'Afrique du Nord (Algérie) et parlées de nos jours sur le sol métropolitain, en particulier par des harkis, précise-t-il, mais aussi par des immigrés récents d'origine algérienne, pourraient déroger à la catégorie des langues de migrants, normalement exclues du champ d'application de la Charte, et se voir attribuer le statut de langues régionales ou minoritaires de France, autant que d'autres, originaires des actuels territoires français. Ces deux rapporteurs en vinrent à faire émerger une catégorie de langues régionales ou minoritaires de migrants, " historiques » car utilisées depuis un certain temps en France et non protégées dans un autre pays. Il

41. "Les "langues dépourvues de territoire" sont exclues de la catégorie des langues régionales ou minoritaires parce qu'elles n'ont pas d'assise territoriale. À d'autres égards toutefois, elles correspondent à la définition de l'article 1 (a), étant des langues traditionnellement employées sur le territoire de l'État par des ressortissants de cet État. Comme exemple de langues dépouvues de territoire, on peut citer le yiddish et le romanes ».

42. "Le texte [de la Charte] concerne les langue régionales ou minoritaires pratiquées "traditionnellement" ; on dit aussi "historiques" (Préambule, § 2). Si cette notion invite à ne pas retenir les langues de l'immigration récente, elle incite au rebours à considérer, du point de vue linguistique, l'histoire de notre pays. De nombreux citoyens des départements français d'Afrique du Nord parlaient l'arabe ou le berbère. Certains, pour des raisons sociales, économiques ou politiques (en particulier les harkis), se sont installés en France métropolitaine, sans cesser d'être des ressortissants français : ils vivent encore, et parlent leurs langues, ou bien leurs descendants ont conservé une pratique bilingue. Cette situation semble correspondre exactement à celle des langues régionales ou minoritaires visées par la Charte. On rappellera que le berbère n'est protégé par aucun pays (il est même menacé) ; on notera que l'arabe parlé en France n'est pas l'arabe classique, langue officielle de plusicurs pays, mais un arabe dialectal, dont certains linguistes pensent qu'il est en passe de devenir une variété particulière, mixte des différents arabes dialectaux maghrébins » (CERQUIGLINI, 1999b). 
convient néanmoins de rappeler que le préambule associe clairement ces langues régionales ou minoritaires « historiques » à l'Europe et que le Rapport explicatif interprète l'adjectif « historiques » comme s'appliquant à des langues «parlées depuis une longue période dans l'État en question " ${ }^{43}$. L'argumentaire français, dans le cas où un projet de ratification serait à nouveau à l'ordre du jour, impliquerait par conséquent de préciser que le berbère, l'arabe dialectal et l'arménien occidental souscrivent à cette condition, il est vrai imprécise quant à ce qui doit être entendu par l'expression « longue période ». Il faut ici rappeler qu'il revient aux États ratificateurs, en vertu de l'article 3.1, d'établir la liste des langues qui entrent dans le champ d'application de la partie III de la Charte, tandis que les dispositions de base contenues dans la partie II sont censées devoir s'appliquer à toutes les langues régionales ou minoritaires qui répondent aux éléments de définition consignés dans le préambule, l'article 1 et le Rapport explicatif. Or, jusqu'à présent, aucun des États ratificateurs n'est sorti du cadre définitoire proposé par la Charte. Ils n'ont pas inclus de langues non européennes, les langues tsiganes ou le yiddish, identifiables à l'Europe, étant pour leur part régulièrement intégrées au titre de l'article 1.c de la Charte comme «langues dépourvues de territoire » par les États concernés dans les déclarations formulées au moment de la signature ou de la ratification.

Un des arguments avancés en France par les opposants à la Charte a justement été qu'elle ne prenait pas en compte les langues des migrants. Les discours qu'ils développent en s'appuyant sur des affirmations de fidélité à une République une et indivisible procèdent, en même temps, d'une attitude négative à l'égard de mesures favorables aux langues régionales ou minoritaires, notamment à leur reconnaissance formelle et à leur accès à des usages publics. L'argument des langues des migrants, véritable problème, prend alors chez eux plutôt l'allure d'un alibi que d'une question. Le Rapport explicatif $(1993, \S 15$, p. 7) fournit une réponse en indiquant que « le CAHLR a estimé que ce problème valait d'être traité séparément, le cas échéant dans un instrument juridique spécifique ». Les langues des migrants, ne pouvant être regroupées sous l'appellation « langues régionales », se trouvent ainsi a priori exclues de cette catégorie ${ }^{44}$. Outre que les États demeurent libres de protéger et de promouvoir les langues de migrants résultant des « récents flux migratoires à motivation souvent économique » (Cerquiglini, 1999b), en s'inspirant éventuellement des schémas d'aménagement linguistique décrits par la Charte, la position française n'en

43. "Les expressions utilisées dans la charte "langues régionales ou minoritaires historiques de l'Europe" (voir deuxième paragraphe du préambule) et "langues pratiquées traditionnellement" dans l'État (article 1, alinéa a) montrent clairement que la charte couvre seulement des langues historiques, c'est-à-dire celles qui sont parlées depuis une longue période dans l'État en question » (Rapport explicatif, 1993, § 31).

44. Pour sa part, Bernard Cerquiglini argumente dans son Rapport la non-éligibilité de certaines de ces langues au titre de la Charte de la façon suivante : « En revanche, des ressortissants français issus, parfois lointainement, de l'immigration parlent encore l'italien, le portugais, le polonais, le chinois, etc. par transmission familiale. Ces langues ne sont pas à retenir ici. Outre que rien ne les menace, elles sont enseignées, comme langues vivantes étrangères, dans le secondaire et le supérieur ». 
est pas moins intéressante en ce qu'elle apporte un élément d'appréciation utile à l'appréhension de ce qui peut être compris, des points de vue sociolinguistique et des droits linguistiques, par « langues régionales ou minoritaires historiques de l'Europe ». Le sujet fut d'ailleurs abordé en 1989 par le CAHLR au cours de sa première réunion. L'emploi du terme « autochtone » fut suggéré et le nombre de générations ayant vécu dans le pays membre du Conseil de l'Europe pour des migrants qui auraient conservé un attachement effectif (pratique, conscience de la langue et demande d'enseignement par exemple, ...) à leur propre langue fut évoqué ${ }^{45}$, mais cette question fut renvoyée à une discussion ultérieure. De fait, le texte de la Charte demeure imprécis sur ces points alors soulevés.

Pour sa part, Guy Carcassonne n'établit pas explicitement de distinction entre « régional » et « minoritaire » et aborde dans les termes suivants l'expression même de « langue régionale », la seule consacrée, jusqu'à présent, en France :

«Sans doute plus discutables, en revanche, sont la dénomination même de langues régionales et l'idée selon laquelle celles-ci seraient attachées à des aires géographiques précisément déterminées. La conception qui veut qu'une langue soit forcément liée à un sol, et ne soit liée qu'à lui, peut être jugée à la fois erronée et dangereuse »(Carcassonne, 1998, p. 5).

Là encore, comme dans le Rapport Giordan de 1982, une contradiction fut soulignée entre le caractère régional revendiqué pour ces langues et le fait que la mobilité sociale les rend également présentes dans la capitale ou d'autres grandes agglomérations urbaines, hors de leurs aires d'origine. De cette façon, si Guy Carcassonne admet par exemple que l'on a plus de chance d'entendre parler alsacien en Alsace qu'en Provence, la langue appartient d'abord au locuteur. Ce dernier se déplace et la langue, selon ce point de vue, est plus celle de ceux qui la parlent que celle d'une région particulière. Cette opinion, qui affirme ne pas être opposée à l'esprit de la Charte, reçue comme un texte ouvert au compromis, présente une option différente au fond quant à la perception de la communication linguistique. Celle-ci s'exerce sur un espace qui, selon l'approche faite par les deux rapporteurs, Guy Carcassonne et Bernard Cerquiglini, n'est pas limité. Si la langue régionale peut posséder à leurs yeux un territoire de référence, historique, mais également où elle est plus présente qu'ailleurs, il n'en demeure pas moins qu'une distance est clairement affichée par rapport à l'idée de territorialisation linguistique. Guy Carcassonne précise clairement son approche :

«Car cette conception, celle d'une localisation régionale d'une langue, est également dangereuse en ceci qu'elle suggère qu'il y aurait une unité, bientôt

45. «Compte tenu des difficultés auxquelles on risque de se heurter pour déterminer si certaines langues sont "parlées traditionnellement sur un territoire donné", certains membres du Comité [CAHLR] proposent d'utiliser le terme "autochtone". Ou bien, on propose de préciser le sens du mot "traditionnellement" en ajoutant, entre crochets, les mots "au moins... générations" " (Comité ad hoc d'experts sur les langues régionales ou minoritaires en Europe (CAHLR), Rapport. Ire réunion, 2-3 octobre 1989, Strasbourg : Conseil de l'Europe, 1989, p. 6). 
une identité, entre ces trois notions qui doivent demeurer très distinctes, que sont un terroir, une langue et un peuple ${ }^{46}$.

Bernard Cerquiglini va dans le même sens en affirmant, dans le fil de l'article 1 de la Constitution de la Vème République, que la langue « régionale » ne saurait être propriété d'une seule section du territoire national ${ }^{47}$. Pour autant, le type d'objet que vise la Charte est bien la langue externe, dont la dimension culturelle est justement indissociable d'un contexte historico-social ${ }^{48}$. Ce dernier correspond, dans son principe à un espace (territoire, aire de parcours) et à un groupe d'intercommunication, même si, de façon secondc, la languc en question peut être conçue comme un bien commun, digne d'être protégé par une société élargie. Et si cette langue n'est pas attribuable d'office au(x) locuteur(s), c'est parce que les mesures préconisées par la Charte prévoient un « appui actif » (Rapport explicatif, 1993, $\S 10$, p. 5) aux langues et non aux individus ou aux groupes en tant que tels. Au-delà de ces considérations, constitutives d'un débat de fond, on notera toutefois que ce qui pourrait aller ici dans le sens d'un statut de personnalité pour ces langues n'est pas mis en évidence dans des textes à caractère légal avec les dispositions concernant leurs droits. Au contraire, c'est plutôt l'aspect territorial qui se détache à travers la notion même de langue régionale, seule utilisée dans le contexte administratif et politique français. C'est aussi celle qui prévaut dans la CELROM, hormis pour les « langues dépourvues de territoire » (cf. article 1.c).

De fait, la désignation « langue régionale » peut se répandre en Europe depuis que plusieurs pays ont signé et parfois ratifié la CELROM. Cette appellation, plutôt employée jusquà présent en France, trouvera là une occasion d'être mieux légitimée. Cela n'empêchera pas l'Espagne, par exemple, de conserver les bases juridiques et sociolinguistiques qu'elle a développées autour de la notion de langue propre ${ }^{49}$. De plus, la Charte est également destinée à des langues offi-

46. Ce qui suit de cette citation mérite également d'être mentionné afin de continuer à expliciter ce raisonnement qui constitue une des illustrations de la position française en matière de langues « régionale » : « Dès lors, en effet, que l'on admettrait, pour prendre un autre exemple, que le breton est la langue de la Bretagne, plutôt que celle de ceux qui la parlent, où qu'ils soient, alors la proposition pourrait être logiquement renversée : la langue de la Bretagne est le breton, d'où il se déduirait que ceux qui habitent celle-là devraient parler celui-ci. Les langues, et leur localisation éventuelle, sont un produit de l'histoire, non de la nature. Elles sont donc liées aux hommes, éventuellement à leurs institutions, non au sol - nul n'a jamais entendu une motte de terre parler français - pas plus qu'au sang - un nouveau né n'a pas de langue : il est seulement détenteur d'une faculté de langage, caractéristique de l'espèce humaine, qui le rend apte à acquérir quelque langue maternelle que ce soit " (CARCASSONNE, 1998, p. 6).

47. « Ce désir d'une assise géographique des langues régionales n'est pas sans contradiction avec l'intention culturelle que la Charte affiche. On peut faire valoir que la territorialisation systématique, issue du romantisme allemand qui inspira la linguistique du XIX ${ }^{\mathrm{e}}$ siècle, s'oppose en outre : - aux principes républicains français, qui tiennent que la langue, élément culturel, appartient au patrimoine national ; le corse n'est pas propriété de la région de Corse, mais de la Nation » (CERQUIGLINI, 1999b, sous "Territoire").

48. Voir, à ce sujet, le commentaire suivant : OYHARÇABAL, 1999, pp. 119-121.

49. Cf. supra, note $\mathrm{n}^{\circ} 21$. 
cielles non étatiques et ne saurait remettre en question des dispositions plus favorables que celles qu'elle propose (cf. article 4.2). Plus largement, la lecture et la mise en œuvre de la Charte témoignent, et témoigneront, de la rencontre et des influences mutuelles entre ses propositions et les acquis conceptuels et empiriques de chacun des pays qui s'engage dans la voie d'une adhésion à elle et de son application.

\section{LECTURES DE LA CHARTE EN CONTEXTE}

\subsection{LA POSITION FRANÇAISE}

La position de la France, à travers les rapports qui furent rédigés en vue de la signature et de la ratification de la Charte, son refus de la ratifier et les débats ayant entouré ces différentes étapes, a été à l'origine d'enseignements utiles à une relecture de ce texte ainsi que de certaines des positions qu'il pouvait susciter. Afin de résumer le blocage actuel, qui résulte de la décision du Conseil constitutionnel du 15 juin 1999 de ne pas ratifier, nous mentionnerons le juriste Jacques Caillosse pour qui ce choix repose plus sur une position de principe, insuffisamment en prise avec une réalité d'ailleurs dévalorisée, que sur une impossibilité juridique ${ }^{50}$. Dans une étude récemment publiée, un autre juriste, Florence Benoît-Rohmer (2001, pp. 23-26), remarque que l'attitude française à propos de la Charte et, en général, à l'égard des langues régionales, est peu respectueuse des standards européens. D'autres juristes, tels Michel Verpeaux ou Ferdinand Mélin-Soucramanien, qui se sont attachés à expliciter le bien-fondé de la décision du Conseil constitutionnel, ont cependant admis qu'elle était basée sur une interprétation restrictive. Ferdinand Mélin-Soucramanien voit dans la Charte une logique différentialiste ${ }^{51}$, alors qu'une approche sociolinguistique, mais aussi, semble-t-il, juridique, pourrait aussi bien relever d'une logique de consensus qui s'y trouve affirmée et développée, à la fois soucieuse

50. « Il s'agit surtout de réactiver le récit fondateur et constitutif de la République, et non de réprimer les langues régionales ou minoritaires. Reste qu'en confirmant l'inconsistance de leur statut juridique, le Conseil constitutionnel conforte une représentation imaginaire du monde dont on doit se demander jusqu'où elle contribue vraiment à resserrer le lien social. La réception de la décision par tous ceux qui ont voulu s'en faire les défenseurs montre combien il est difficile de ne pas y voir une dévalorisation en actes des langues mineures parce que périphériques " (CAILlosSE, 2000, p. 108).

51. Où finit la justification et où commence la crainte déraisonnable, voire l'amalgame, lorsqu'on lit le commentaire suivant, inspiré, il est vrai, par la décision du Conseil constitutionnel au sujet de la Charte : "Sauf à se déjuger et à rompre avec une ferme interprétation des principes d'indivisibilité de la République et d'unicité du peuple français partagée avec le Conseil d'État, le Conseil pouvait difficilement ignorer la logique différentialiste exprimée dans la Charte européenne des langues régionales ou minoritaires. Il faut en effet être bien conscient des conséquences possibles d'une reconnaissance juridique de la liaison entre une langue et un territoire. Les exemples du conflit armé en ex-Yougoslavie ou de la crise identitaire que traverse actuellement la Belgique démontrent à l'envi que la différenciaition linguistique contient en germe la ségrégation et la fragmentation de l'État » (MÉlin-SOUCRAMANIEN, 1999, pp. 994-995). 
de la richesse culturelle de l'Europe et d'une gestion maîtrisée de cette dernière, sans préjudice pour les situations acquises par les langues officielles d'État ou de plus grande diffusion.

À la suite des trois rapports rédigés à la demande du Gouvernement par Bernard Poignant (1998), maire de Quimper, en juillet 1998, par Guy Carcassonne (1998), juriste, en septembre 1998, et par Bernard Cerquiglini (1999b), linguiste, en avril 1999, la France apposa sa signature à la CELROM, le 7 mai 1999, en l'assortissant d'une Déclaration interprétative destinée à accompagner un futur instrument de ratification ${ }^{52}$. Cettc déclaration présentait certaines restrictions portant notamment sur l'emploi du terme « groupe », sur l'obligation d'employer le français dans les relations avec les administrations et les services publics, sur le caractère facultatif de l'enseignement des langues régionales ou minoritaires et sur le fait que seules les versions en français des textes législatifs, par ailleurs accessibles dans les langues régionales, fissent juridiquement foi. Suivait la liste des trente-neuf dispositions de la partie III de la Charte que la France se serait engagée à appliquer au moment d'une future ratification. Les choix, encadrés par les contraintes souples inscrites dans l'article 2 alinéa 2, s'inscrivaient principalement dans les domaines de l'enseignement (article 8), des médias (article 11), des « activités et équipements culturels » (article 12) et de la «vie économique et sociale » (article 13). Le 20 mai de la même année, le Président de la République saisit le Conseil constitutionnel en application de l'article 54 de la Constitution afin de savoir si, « compte tenu des engagements que la France entend souscrire dans la partie III de cette convention, la ratification de la Charte européenne des langues régionales ou minoritaires, signée à Budapest le 7 mai 1999, doit être précédée d'une révision de la Constitution ». Le 15 juin suivant, le Conseil constitutionnel s'opposa à la ratification, dans sa décision $n^{\circ}$ 99-412 DC, estimant que la Charte comportait des clauses contraires à la Constitution. Cette instance ne fonda pas son rejet sur l'opportunité des engagements choisi par le Gouvernement dans la partie III de la Charte - il en fallait un minimum de trente-cinq, et le juriste Guy Carcassonne avait proposé qu'il en soit retenu cinquante et un, ce qui correspondait à la moyenne des dispositions admises par les Parties - mais estima que certaines de celles que contenaient le préambule et la partie II ne pouvaient être recevables en droit français.

Au départ, la France hérite d'une tradition de défiance, sinon d'opposition, aux autres langues de France que le français, consacré depuis longtemps seule langue nationale et officielle, l'unitarisme dans ce domaine étant censé protéger le pays de velléités séparatistes ${ }^{53}$. L'usage des langues dites régionales, perçues comme un terreau possible pour le développement de tendances centrifuges ou

52. Seules la France et la Slovaquie ont déposé à ce jour une déclaration au moment où elles ont signé la Charte. Elles n'ont pas encore ratifié ce texte.

53. Les opposants les plus convaincus à la ratification de la CELROM par la France, dont le ministre de l'Intérieur d'alors, Jean-Pierre Chevènement, virent un risque de balkanisation dans toute tentative visant à octroyer un statut légal aux langues régionales ou minoritaires. 
irrédentistes et non comme une richesse culturelle, y relève par ailleurs, comme la religion, de l'exercice privé d'une liberté publique. Seul le français a juridiquement accès à l'exercice public. En dehors de l'enseignement où des efforts ont été faits au cours des deux dernières décennies, ce sont uniquement des aménagements symboliques ou peu importants, ainsi que des tolérances, qui semblent envisageables. Il conviendra de rappeler que le Gouvernement, à deux reprises, durant les dernières décennies, a ratifié des conventions internationales en les assortissant de réserves concernant les articles où il était fait mention de la notion de minorité et, en l'occurrence, de langue minoritaire. Nous nous référerons notamment ici au Pacte international relatif aux droits civils et politiques des Nations Unies de 1966, pour lequel une des réserves fut émise sur l'article 27 lors de l'adhésion en 1981, et la Convention des Nations Unies relative aux droits de l'enfant de 1990, pour laquelle une autre réserve porta sur l'article 30 lors de la ratification la même année. Deux années plus tard, la loi constitutionnelle du 25 juin 1992, prévue pour protéger le français de la concurrence de l'anglais avant la ratification du traité de Maastricht ${ }^{54}$, stipulait que « La langue de la République est le français ». Aucun amendement reconnaissant les langues régionales et leur garantissant une protection minimale ne fut retenu, le ministre d'alors ayant par ailleurs assuré, au cours des débats, que cette loi ne saurait leur être préjudiciable. Depuis son insertion en 1993 dans le corps de la Constitution, où elle ouvre le nouvel article 2 , cette disposition n'a pourtant cessé d'être alléguée par les instances juridictionnelles supérieures de l'État, Conseil d'État et Conseil constitutionnel, contre, notamment, l'accès des langues régionales à des usages publics, ainsi que nous avons déjà eu l'occasion de le signaler.

En ce qui concerne la CELROM, et sans que ces antécédents exercent une influence forcément déterminante sur les considérants du Conseil constitutionnel (Carcassonne, 1998, pp. 46-48), ce dernier s'est appuyé sur sa propre jurisprudence et sur celle du Conseil d'État. Celui-ci avait rendu le 24 septembre 1996 un avis défavorable à l'adhésion de la France à la Charte en se fondant, notamment, sur le premier alinéa de l'article 2 de la Constitution (cf. supra). La jurisprudence du Conseil constitutionnel s'appuyait sur trois décisions. La décision $n^{\circ}$ 91-290 DC du 9 mai 1991, "sur la loi portant statut de la collectivité territoriale de Corse », avait rejeté la notion de «peuple » corse et l'enseignement obligatoire de la langue corse au nom des principes d'indivisibilité de la République et d'égalité des citoyens devant la loi. La décision n ${ }^{\circ}$ 94-345 DC du 29 juillet 1994, qui portait « sur la loi relative à l'emploi de la langue française », avait en revanche constaté l'usage des langues régionales ainsi que la possibilité d'utiliser des traductions pour des textes à caractère légal et administratif (considérant 7). La décision $n^{\circ}$ 96-373 du 9 avril 1996, s'appliquant à la loi organique portant statut d'autonomie de la Polynésie française, avait défini, en application de l'article 2 alinéa 1 de la Constitution, les règles d'usage linguistique dans les relations avec les administrations et services publics. Les particuliers ne peuvent « se prévaloir », dans ces cas, « d'un droit à l'usage d'une langue autre que le

54. Signé le 7 février 1992 à Maastricht, il fut ratifié par la France le 20 septembre suivant. 
français, ni être contraints à tel usage », mais, fut-il rappelé (cf. supra, décision $n^{\circ}$ 94-345 DC), « l'article 2 de la Constitution n'interdit pas l'utilisation de traductions ». Néanmoins, dans le troisième considérant de la partie de sa décision de 1999 portant sur la conformité de la Charte à la Constitution, le Conseil constitutionnel déclara que les dispositions du préambule et de la partie II de la Charte étaient « également contraires au premier alinéa de l'article 2 de la Constitution en ce qu'elles tendent à reconnaître un droit à pratiquer une langue autre que le français non seulement dans la "vie privée" mais également dans la "vie publique", à laquelle la Charte rattache la justice et les autorités administratives et services publics ». Cela concernait le droit imprescriptible de pratiquer une langue régionale ou minoritaire dans la vie privée ou publique, énoncé dans le préambule de la Charte, et, en définitive, la référence faite aux groupes de locuteurs de ces langues et aux territoires dans lesquels elles sont utilisées (article 7) comme autant d'entités suffisamment identifiables d'un point de vue administratif pour justifier de mesures d'aménagement linguistique. Le danger que ces dispositions présentaient aux yeux du Conseil constitutionnel sur un point aussi important, « en ce qu'elles tendent à », était-il fondé ? L'irrévocabilité d'une conclusion reposant, au moins dans ce considérant, sur ce qui revenait à être une supposition paraît effectivement s'appuyer sur une position de principe, pour reprendre l'opinion de Jacques Caillosse signalée plus haut. Admettant implicitement l'applicabilité de la Charte en France, le Conseil crut peut-être qu'elle risquât d'affaiblir des principes, érigés par voie de conséquence en remparts. Guy Carcassonne avait en outre rappelé dans son rapport que le préambule, en droit international, est un exposé des motifs et qu'à ce titre, « il n'a pas force obligatoire, mais constitue, au plus, un élément d'interprétation du traité ou de la convention » (Carcassonne, 1998, pp. 45-46). Cela réduisait la force de cette imprescriptibilité pourtant évidente dans une approche sociolinguistique de l'aménagement linguistique.

La décision française, qui ne témoigne pas d'une unanimité politique, a présenté l'intérêt de poser indirectement la question du seuil d'un aménagement linguistique de langues peu répandues, fondé, comme cela avait été approuvé dans le rapport Carcassonne, sur l'absence de discrimination, sur le caractère facultatif de mesures sélectionnées dans les domaines de l'enseignement ainsi que dans les administrations et les services publics et sur un accès minimal à des usages à caractère officiel, au moins à travers des traductions de textes en français faisant seuls foi. Certes, il y a eu des avancées significatives dans l'enseignement des langues régionales en France, surtout depuis le milieu des années quatrevingt, mais concevoir qu'une langue doive être cantonnée à un usage privé revient à lui dénier l'accès, aménagé au besoin, à des usages formels, pourvoyeurs du prestige minimal dont toute langue doit pouvoir bénéficier dans un cadre démocratique. Or la reconnaissance légale d'une langue, globale et ne couvrant pas seulement l'enseignement, constitue une source significative de prestige afin que des représentations positives suscitent l'apparition d'une loyauté consciente à son endroit (Viaut, 1999). L'efficience d'un lien dynamique entre prestige et loyauté représente une des conditions favorables à l'utilisation d'une 
langue. Les propositions que fait la Charte tendent justement à assurer un seuil minimum en deçà duquel des langues en danger ne profiteraient pas de conditions suffisantes suscitées par ce lien.

Au-delà du caractère quasi sacré, car sans appel, de ce qui émane du Conseil constitutionnel, il convient de noter que cette décision remet en cause les fondements mêmes de la Charte. Pour l'heure, ce qui aurait pu apparaître, à l'échelon des grandes références, comme un apport positif dans le débat pour les droits linguistiques en présentant une conception revisitée de l'universalisme à la française, plus intégrateur que niveleur et soupçonneux à l'égard des individualités culturelles, n'a pas été. Cette réaction est et restera minoritaire au sein du Conseil de l'Europe et, encore plus, parmi les pays qui sont en même temps membres de l'Union européenne. L'implication sociale de la position française est ainsi exposée par Jacques Caillosse :

« Il s'agit surtout de réactiver le récit fondateur et constitutif de la République, et non de réprimer les langues régionales ou minoritaires. Reste qu'en confirmant l'inconsistance de leur statut juridique, le Conseil constitutionnel conforte une représentation imaginaire du monde dont on doit se demander jusqu'où elle contribue vraiment à resserrer le lien social. La réception de la décision par tous ceux qui ont voulu s'en faire les défenseurs montre combien il est difficile de ne pas y voir une dévalorisation en actes des langues mineures parce que périphériques » (Caillosse, 2000, p. 108).

Le Rapport explicatif spécifie pourtant que les langues, et non les individus ou les groupes, sont objet du droit dans cette convention. En outre, les « territoires » auxquels il est fait référence ne contreviennent pas forcément à la jurisprudence française de l'égalité par la différenciation (cf. supra). L'enseignement des langues régionales en France est d'ailleurs territorialisé pour l'essentiel en fonction, en principe, des demandes et, surtout, des réalités administratives et linguistiques. La possibilité d'accès, incontournable d'un point de vue sociolinguistique, des langues régionales ou minoritaires à la « vie publique » fut rejeté, nous l'avons vu, comme non conforme à l'article 2 de la Constitution malgré, même, les dispositions minimales possibles retenues dans la partie III de la Charte en matière de traduction. Les indications rappelées dans le rapport rédigé par Guy Carcassonne sur le caractère facultatif d'un tel accès et la Déclaration interprétative accompagnant la signature de la Charte par le gouvernement ${ }^{55}$ n'y firent rien. À la suite de cela, le Président de la République refusa d'engager la révision constitutionnelle demandée par le Premier ministre. Il y a peu de

55. «Le Gouvernement de la République interprète l'article 7.1 paragraphe d ["la facilitation et/ou l'encouragement de l'usage oral et écrit des langues régionales ou minoritaires dans la vie publique et dans la vie privée"] et les articles 9 et 10 comme posant un principe général n'allant pas à l'encontre de l'article 2 de la Constitution selon lequel l'usage du français s'impose aux personnes morales de droit public et aux personnes de droit privé dans l'exercice d'une mission de service public, ainsi qu'aux usagers dans leurs relations avec les administrations et services publics » (Déclaration formulée par la France lors de la signature de la Charte européenne des langues régionales ou minoritaires, I.2). 
chance pour qu'il soit dès lors question de mettre en œuvre une autre procédure de révision aux termes de l'article 89 de la Constitution. Cette configuration « à la française $\gg s^{\prime} e s t$, pour certains, refermée sur elle-même ${ }^{56}$.

Le Conseil constitutionnel a craint que l'adhésion aux principes généraux de la Charte, contenus dans le préambule et la partie II, n'entrainât une reconnaissance de l'existence de groupes linguistiques, même s'il est clair qu'ils ne sont pas des objets du droit pour la Charte. En outre, l'argument opposé de l'unicité du peuple français inscrite dans l'article 1 de la Constitution doit être approché en tenant compte du fait que ce type de déclaration existe également dans d'autres constitutions de pays européens. Comme le rappelle Michel Verpeaux, la Constitution de l'Allemagne, pays fédéral, se réfère à un seul peuple allemand et celle de l'Espagne, pays unitaire largement décentralisé en État dit « des autonomies », renvoie également à l'unité du peuple espagnol (Verpeaux, 1999, pp. 599-600). Rappelons-le, la Charte s'inscrit dans une tradition d'un demisiècle maintenant, depuis la Convention de sauvegarde des droits de l'homme et des libertés fondamentales (cf. supra), qui faisait des « personnes » l'objet du droit. La Charte, quant à elle, met les langues en avant.

Le Conseil constitutionnel n'a pas cru adéquat de s'appuyer sur la Déclaration interprétative qui avait accompagné la signature de la Charte par la France et qui, en droit, ne peut se limiter qu'à préciser la signification que l'État attribue au texte qu'il signe ou ratifie. En revanche, relevant les passages du préambule et de l'article 7 - spécialement les alinéas 1 et 4 -qui contrevenaient à son sens à certains des principes du droit français et qui avaient fait l'objet de cette Déclaration interprétative, le Conseil n'a pas recouru à l'article 21 de la Charte qui lui permettait de « formuler une ou plusieurs réserves aux paragraphes 2 à 5 de l'article 7 ». Or il est justement fait explicitement appel, dans ces paragraphes, à la notion de groupe, tempérée par le Rapport explicatif, comme nous l'avons évoqué plus haut. Cette voie, qu'un seul pays, la Croatie, jusqu'à présent, a utilisée ${ }^{57}$, aurait au moins concerné directement l'article 7 alinéa 4 (cf. supra) visé par la décision du Conseil constitutionnel. Ce type d'argumentation aurait été inutile si le Conseil avait accepté celle qu'avait développée la Déclaration interprétative qui précisait que « l'emploi du terme de "groupes" de locuteurs ne confère pas de droits collectifs pour les locuteurs des langues régionales ou minoritaires ${ }^{58}$. Cela allait dans le sens des assertions déjà claires de la Charte

56. Deux essais polémiques, publiés en 1999 et 2001 , illustrent la radicalisation de certaines prises de position à l'égard de la Charte en France. Leurs titres sont eux-mêmes parlants, avec les « féodalités » et une « guerre des langues » qui serait censée évoquer un risque de retour à des relations franco-allemandes du type de celles qui existèrent à des moments sombres de l'histoire. Il s'agit des ouvrages de René Andrau (1999) et d'Y Yonne Bollmann (2001).

57. La réserve portant sur le paragraphe 5 écarte ainsi du champ d'application de la Charte les « langues dépourvues de territoire», telles que, notamment, celles des Tsiganes. Il en résulte que seules les dispositions générales du préambule peuvent s'appliquer à ces dernières langues que l'article 1 comprend parmi les langues régionales ou minoritaires.

58. In : Déclaration formulée par la France lors de la signature de la Charte européenne des langues régionales ou minoritaires, I.1. 
et de son Rapport explicatif à ce propos. L'opinion émise par Alain Renaut au sujet de la Charte dans la revue Comprendre rappelle une relation essentielle entre l'individu, la langue et le groupe, correspondant à l'approche traditionnelle libérale des droits de l'homme, aussi bien en ce qui concerne le primat des droits individuels que pour valider les droits linguistiques d'individus « envisagés dans leur appartenance à un espace culturel et linguistique dont, dans la représentation qu'ils se forgent de leur propre identité, ils considèrent qu'ils le partagent avec d'autres » (Renault, 2000, pp. 395-396). Alain Renaut rappelle de surcroît que « la reconnaissance de droits linguistiques ainsi compris s'inscrirait pleinement dans le cadre d'une tentative plus vaste pour adjoindre à la Déclaration universelle des droits de l'homme un avenant relatif aux droits culturels de l'individu » (ibid, p. 396). Florence Benoît-Rohmer (2001, pp. 28-29) va dans le même sens en estimant que les droits de l'homme et de la citoyenneté ne peuvent plus être réduits à leur dimension politique et doivent être étendus au champ du culturel. La langue existe en tant que telle à travers le groupe auquel elle fournit un lien indispensable à sa cohérence et l'individu, en choisissant de s'y agréger ou pas, en passe par la langue ou par l'acceptation de se référer à elle comme à l'une de ses valeurs. Mais les groupes et les pratiques ne sont pas forcément monolithiques. Ils peuvent l'être à travers les représentations ou les conceptions que s'en font certains. Les unes et les autres sont plus souvent et, réellement, enchâssées. C'est à la gestion de ce type de réalité que la Charte peut précisément contribuer. À partir de là, l'identité linguistique peut être perçue selon une conception unitariste et hégémonique, ou bien selon une déclinaison ou une articulation des pratiques et des attachements linguistico-culturels.

Le débat français a fait resurgir sur la place publique des questions qui avaient déjà été soulevées lors de la mise au point de la Charte. Les analyses et les discussions qui en ont résulté sont de nature à permettre de préciser encore les termes de cette convention et, en cela, elles sont sans doute utiles. Les opposants à la Charte les plus déterminés, en attendant qu'ils assouplissent peut-être leurs positions, ont tendance en France à se raidir, comme nous l'avons déjà souligné, et à se laisser aller à de périlleux amalgames ${ }^{59}$. Ils lient leur combat contre la Charte à celui qu'ils mènent contre un développement de l'intégration européenne et contre l'Europe des régions dont ils estiment qu'elle pourrait peser trop lourd par rapport à celle des États. Au-delà de ces considérations partisanes, on peut se demander si la décision française fera école auprès d'autres partenaires du Conseil de l'Europe ou si elle résume - et clôt peut-être - un combat d'arrière-garde. Pour l'heure, les adhésions à la Charte sont plus nombreuses,

59. Ainsi, les deux citations qui suivent prêtent des intentions funestes aux nouveaux ennemis d'une France persécutée par une Europe des régions sous influence germanique : « Si la France ratifiait la Charte des langues, elle offrirait enfin à ses adversaires ethnistes le signe de reddition qu'ils attendent depuis si longtemps »; «Car ce texte est une machine de guerre, qui a pour cible principale la France, et sa conception non ethnique de la citoyenneté. Vouloir l'ignorer revient à s'y exposer sans défense. C'est reproduire aujourd'hui, simplement sous une autre forme, le courant pacifiste français de l'entre-deux-guerres, qui a révélé son irréalisme en juin 1940 " (Boll.manN, 2001 , pp. 39 et 64 ). 
sans toutefois que la majorité des pays membres de cette organisation ait encore été atteinte. On relèvera cependant que, le 20 juillet 1999, la Cour constitutionnelle roumaine rendit une décision sur la compatibilité de la Charte avec sa Constitution qui fut différente sur le fond de celle qu'avait prise le Conseil constitutionnel français un mois auparavant. La Roumanie se définit comme un pays unitaire, et le roumain est la seule langue officielle d'État. Soucieuse de s'assurer que la CELROM ne contrevenait pas à des grands principes proches de ceux que le Conseil constitutionnel français avait choisis comme références, la Cour roumaine admit simplement que le texte de la Charte répondait à ses attentes (Commenté in Benoît-Rohmer, 2001, pp. 14-15). L'on finit par se demander si, dans la position que la France a pris la responsabilité d'illustrer, c'est uniquement le contenu de la Charte qui est en cause ou bien aussi le fait de porter sur la place publique un sujet tabou dont le règlement finirait par relever autant du droit que de l'ethnologie et de la psychologie collective. Il est ainsi frappant de constater que l'une des différences qui apparaissent entre les déclarations accompagnant les ratifications, qui adoptent plutôt un ton neutre et gestionnaire, et celle que déposa la France au moment de sa signature provient du recours par cette dernière à des principes républicains destinés à exercer une fonction protectionniste à l'égard de la langue française. Un décalage paraît exister entre les réticences ou la frilosité d'une partie de la classe politique et l'opinion publique si l'on en croit des résultats de l'institut de sondage IFOP ${ }^{60}$ qui signalent l'existence d'un courant majoritaire en faveur de la CELROM en France. La progression de l'intégration européenne aidant, rien n'est donc $a$ priori scellé, mais cela peut prendre du temps. En attendant, la partie des mesures de la partie III de la Charte que la France avait annoncé retenir au moment de la signature est plus ou moins déjà en application, essentiellement dans l'enseignement où elles ne sont encore proposées qu'à un nombre réduit d'élèves. Malgré cela, la demande est en croissance dans ce domaine ${ }^{61}$, surtout dès qu'une offre est proposée.

60. Le Comité d'Alsace-Lorraine du Bureau européen pour les langues moins répandues a fait réaliser par l'IFOP, les 6 et 7 avril 2000, une étude sur l'opinion des Français au sujet de ratification de la Charte. Il en ressort que $82 \%$ des personnes interrogées se sont déclarées favorables à cette adhésion. Ce pourcentage se répartit en $27 \%$ de « tout à fait favorables » et en $55 \%$ de «plutôt favorables ». En comparaison, l'IFOP avait procédé en avril 1994 à un sondage intitulé Les Français et les langues régionales, dans lequel la dernière question portait également sur l'adhésion à la Charte. Quatre possibilités de réponse avaient été proposées : « plutôt favorable », «plutôt opposé ", " ni l'un ni l'autre » et " ne se prononce pas ». $77 \%$ des enquêtés avaient répondu «plutôt favorable».

61. Cf. les chiffres fournis par Jack Lang, ministre de l'Éducation nationale, le 25 avril 2001 sur «Les nouvelles orientations pour le développement de l'enseignement des langues régionales » (site internet : http://www.education.gouv.fr/discours/2001/regionlang.htm). Il signala, entre autres chiffres, celui d'une progression, au cours des deux dernières années, de $25 \%$ du nombre d'élèves suivant un enseignement bilingue français/langue régionale dans le premier degré et de $100 \%$ dans le second degré. 


\subsection{UNE RÉFÉRENCE POUR L'AVENIR DU TRAITEMENT DES DROITS LINGUISTIQUES}

Il apparaît, par conséquent, que des interprétations particulières se dégagent des déclarations accompagnant les signatures ou, surtout, les ratifications de la CELROM. D'autres nuances ou lectures, provenant de pays et d'expériences divers, peuvent finalement contribuer pour l'avenir à affiner ces différentes définitions. D'une part, les Déclarations utilisent, jusqu'à présent, régulièrement la désignation « langue régionale ou minoritaire ». L'appellation « langues officielles moins répandues » n'a jusque-là été employée que dans la déclaration suisse de 1997 au sujet du romanche et de l'italien. La Finlande, dans la déclaration de ratification qu'elle déposa en 1994, employa, elle, l'expression proche de « langue officielle la moins couramment utilisée » à propos du suédois. Il en résulte, dans cette lecture finnoise de la Charte, une distinction entre la notion de « langue régionale ou minoritaire », qui est appliquée au sami, et celle de « langue officielle moins couramment utilisée », qui est réservée au suédois. Pourtant, aux termes de l'article 1.a de la CELROM, la première notion pouvait s'appliquer aux deux langues.

La République fédérale d'Allemagne a fait une interprétation particulière de la notion de langue régionale ou minoritaire dans la Déclaration qu'elle a transmise au Secrétariat général du Conseil de l'Europe en 1998 pour en préparer la ratification. Ce pays a établi une distinction entre langue régionale et langue minoritaire, réservant la première dénomination au bas allemand et la deuxième à six autres langues ${ }^{62}$. Il nous paraît se dégager de ce choix que la notion de langue régionale serait, dans cet esprit, applicable à une langue linguistiquement proche de la langue officielle d'État, comme pourrait l'être une des langues d'ö̈l par rapport au français « national et standard » (cf. supra). On a déjà vu plus haut que, dans son rapport, Guy Carcassonne avait proposé de distinguer les deux notions sur la base d'autres arguments.

Autre cas de figure : dans l'Instrument de ratification que l'Espagne a déposé le 9 avril 2001, aucune langue n'est nommée. Deux catégories de langues sont distinguées. Celles qui sont officielles dans les Statuts d'autonomie des Communautés administratives concernées se voient confirmer une protection maximale dont elles jouissaient généralement déjà. Aux langues protégées (asturien et aragonais, a priori) « s'appliqueront toutes les dispositions de la Partie III de la Charte qui peuvent raisonnablement s'appliquer conformément aux objectifs et principes établis à l'article $7 \gg 63$. La nomination aurait en effet pu être exploitée comme la légitimation, dans un sens ou dans un autre, de langues dont l'autonomie est discutée. Il en allait essentiellement de la notion de

62. Ces autres langues sont les suivantes : danois, haut sorabe, bas sorabe, frison septentrional, frison saterois, rom.

63. In : Liste des déclarations du traité $n^{\circ} 148$ Charte européenne des langues régionales ou minoritaires. Historique complet au : 20/06/01. Adresse internet : http://conventions.coe.int/treaty/ FR/DeclareList.asp?NT $=148 \& \mathrm{CM}$. 
« valencien » par rapport à celle de « catalan ». Le valencien est classiquement décrit comme faisant partie du continuum catalan, intégré à l'aire du catalan occidental, mais fait par ailleurs l'objet d'une particularisation dans le but de l'instituer comme langue différente du catalan. Les protagonistes (Académie de la culture valencienne, ...) de cette tendance refusent le rôle référentiel exercé par le standard de Catalogne et les normes dites de Castelló (adaptation à la variété valencienne en 1932 des règles orthographiques de l'Intitut d'études catalanes). Le débat demeure ainsi interne. L'État, responsable de l'adhésion à la Charte, renvoie, sans prendre la responsabilité de les nommer ${ }^{64}$, aux langues effectivement retenues par les divisions administratives (Communautés autonomes) espagnoles responsables de la politique linguistique. La Déclaration accompagnant la ratification allemande avait, elle, fourni les détails des dispositions choisies, Land par Land. Ce qui peut être attendu de l'application de l'article 3 alinéa 1 de la Charte, qui demande que soit spécifiée, dans l'Instrument de ratification, chaque langue régionale ou minoritaire ou chaque langue officielle moins répandue devant faire l'objet de la partie III, peut recevoir une réponse maximale ou minimale. Les soixante-quinze langues nommées dans le Rapport Cerquiglini pour la France, même si l'adhésion, au moins momentanément, n'a pas abouti, constituent peut-être un extrême porteur de ses propres enseignements. La position adoptée par l'Espagne est à l'opposé, et la Charte est finalement indirectement utilisée pour contribuer à neutraliser des tensions internes.

L'Autriche, qui a déposé son Instrument de ratification le 28 juin 2001 , a introduit dans la Déclaration l'accompagnant une interprétation supplémentaire du lien langue-territoire. Le document se compose de deux parties. La première est destinée à des langues (croate, slovène, hongrois) territorialisées dans des Länder où elles représentent le plus de locuteurs concernés. La deuxième s'applique, d'une part, à les langues très peu répandues dans des Länder où elles sont traditionnellement implantées (le rom dans le Burgenland et le slovène en Styrie) et, d'autre part, à des langues implantées dans l'agglomération viennoise, hors de leur aire d'origine. Sont ainsi concernés le tchèque, le hongrois et le slovène. En dehors du slovène et, dans une moindre mesure, du hongrois, qui sont implantés dans des régions particulière (Carinthie, Burgenland), le tchèque et le hongrois sont par ailleurs des langues européennes liées à une immigration et à des échanges qu'il faut replacer dans le contexte historique proprement autrichien de l'ancien Empire austro-hongrois. Cette configuration autrichienne contribue ainsi à relativiser la notion de territoire linguistique. Elle nous renvoie utilement aux questions évoquées en France dans le Rapport Giordan au sujet de la présence des langues régionales dans les grandes concentrations urbaines. Elle nous rappelle également les questions soulevées en 1989 par le Comité ad

64. Dans ce cas précis, la situation est également compliquée du fait que les institutions politiques et académiques valenciennes désignent par «valencien » ce qu'elles conçoivent comme faisant partie de ce continuum catalan dont elles ont intégré, pour la plus large part, les normes codificatrices élaborées sur la base directrice du catalan de Catalogne. 
hoc d'experts sur les langues régionales ou minoritaires en Europe au sujet du degré d'historicité de langues de migrants (cf. supra), ainsi que le caractère « historique » de langues, dans ce cas, « traditionnellement pratiquées sur le territoire de l'État » (article 1.c).

Les adhésions à venir feront peut-être apparaître d'autres conceptions et des tendances pourront ainsi se détacher plus nettement encore. Les cas spécifiques n'en seront que plus évidents. En attendant, certaines des conceptions qui se sont fait jour jusqu'à présent, dans le cadre ou la perspective de l'adhésion à la Charte, intègrent explicitement la notion de territoire (cf. Allemagne, Autriche, France par exemple). D'autres, plus nombreuses, sont discrètes à cet égard dans la mesure où les langues qu'elles visent sont d'abord présentées à l'aide de formulations neutres comme : " la Charte s'appliquera aux langues $[\mathrm{x}, \mathrm{y} \ldots]$ » (cf. Déclaration hongroise) ou « les langues $[\mathrm{x}, \mathrm{y}, \ldots]$ sont considérées comme

des langues régionales ou minoritaires au sens de la Charte européenne... » (cf. Déclaration slovène). Il est enfin un cas particulier qui est apparu à l'occasion de la signature de la Charte par l'Azerbaïdjan en décembre 2001. Dans la Déclaration consignée dans une Note verbale, il est en effet dit que « La République d'Azerbaïdjan déclare qu'elle n'est pas en mesure de garantir l'application des dispositions de la Charte dans les territoires occupés par la République d'Arménie ». La question posée est celle de l'application du texte dans un territoire occupé ou en proie à des troubles de nature à perturber l'application du droit. Or l'Arménie a ratifié la Charte en janvier 2002. Ces faits nous suggèrent deux observations : parmi les langues que l'Arménie a annoncé protéger ne figure pas l'azéri pourtant réellement présent dans ce pays. Par ailleurs, l'arménien est présent de façon compacte dans le Haut-Karabakh qui a relevé jusqu'à présent de l'Azerbaïdjan, mais qui est occupé aujourd'hui par l'Arménie. Une des raisons du litige est liée aux droits culturels et linguistiques des uns et des autres. Le mot « arménien » ne figure pas dans la Déclaration azerbaïdjanaise (peut-être apparaîtra-t-il dans un ultérieur et éventuel instrument de ratification ?), pas plus que le mot « azéri » dans l'instrument arménien. Or la question linguistique est, dans ces cas, on ne peut plus liée au territoire. Il est alors regrettable que son traitement n'ait sans doute pu être avancé de manière un peu plus résolue pour éventuellement contribuer à neutraliser les tensions entre ces deux pays.

\section{RÉFÉRENCES BIBLIOGRAPHIQUES}

ANDRAU René (1999), Les féodalités reviennent : réflexions sur la Charte européenne des langues régionales ou minoritaires, S. I. : Bruno Leprince éditeur, 126 p.

Aracil Lluís V. (1982), Papers de sociolingüística, Barcelona : La Magrana, 249 p.

ARQUINT Romedi (1998), "Les langues régionales ou minoritaires et les problèmes d'éducation", in Conférence internationale sur la Charte européenne des langues régionales ou minoritaires (Conférence organisée par la Direction de l'environnement et des pouvoirs locaux du Conseil de l'Europe, Strasbourg, 26-27/03/1998), Strasbourg : Conseil de l'Europe, Langues régionales ou minoritaires, $\mathrm{n}^{\circ} 1$, pp. 18-23. 
BENOÎT-ROHMER Florence (2001), "Les langues officieuses de la France", Revue française de droit constitutionnel, $\mathrm{n}^{\circ} 45$, pp. 3-29.

BlaIR Philip (1998), "Présentation de la Charte européenne des langues régionales ou minoritaires", in Conférence internationale sur la Charte européenne des langues régionales ou minoritaires (Conférence organisée par la Direction de l'environnement et des pouvoirs locaux du Conseil de l'Europe, Strasbourg, 26-27/03/1998), Strasbourg : Conseil de

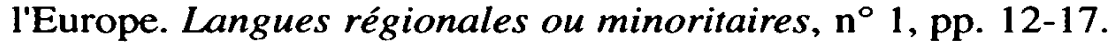

BOLLMANN Yvonne (2001), La bataille des langues en Europe, Paris : Bartillat, 175 p.

CAILlosse Jacques (2000), "L'ordre de la langue", Pouvoirs locaux. Les cahiers de la décentralisation, $\mathrm{n}^{\circ} 46$, III, septembre, pp. 100-108.

Calvet, Louis-Jean (1993), L'Europe et ses langues, Paris : Plon, 238 p.

CARCASSONNE Guy (1998), Étude sur la compatibilité entre La Charte européenne des langues régionales ou minoritaires et la Constitution, Rapport réalisé à la demande du Premier ministre, septembre, $130 \mathrm{p}$.

Cerquiglini Bernard (1999a), "La Charte européenne des langues et cultures régionales ou minoritaires", in Langues et cultures régionales de France. État des lieux, enseignement, politiques, Actes du colloque (11-12.06.1999, Université de Paris V - René Descartes), Paris : L'Harmattan, pp. 107-111.

CERQUIGLINI Bernard (1999b), Les langues de France. Rapport au Ministre de l'Éducation nationale, de la recherche et de la technologie et à la Ministre de la culture et de la communication, Avril, 8 p. (http://www.culture.gouv.fr/culture/dglf/, sous "Les langues de France").

Chapalain Ana-Vari (1995), "Bilan des actions en faveur de la Charte européenne", in Cinquième rencontre interrégionale Langues et culture spécifiques (22-24 juin 1995), Ustaritz : Institut Culturel Basque, pp. 85-90 + annexes.

Clotet I MIRÓ Maria Angels (1994), "La carta europea de las lenguas regionales o minoritarias", Revista de Instituciones Europeas, pp. 529-562.

Combat laïque (Comité Laïcité République Vallée de la Dordogne) (1999), $n^{\circ} 4$ et 5 , décembre.

Conférence internationale sur la Charte européenne des langues régionales ou minoritaires, Conférence organisée par la Direction de l'environnement et des pouvoirs locaux du Conseil de l'Europe (Strasbourg, 26-27/03/1998), Strasbourg : Conseil de l'Europe, Langues régionales ou minoritaires, $\mathrm{n}^{\circ} 1,1998,61 \mathrm{p}$.

COSERIU Eugenio (1988), " "Historische Sprache » und «Dialekt »", in Studia in honorem Eugenio Coseriu. Band I. Schriften von Eugenio Coseriu (1965-1987), eingeleitet und herausgegeben von Jörn Albrecht, Tübingen : Gunter Narr, pp. 45-61

CoYos Jean-Baptiste (1999), "Nommer les langues, un enjeu. Le cas des langues dites 'régionales' de France", in Langues et cultures régionales de France. État des lieux, enseignement, politiques, Actes du colloque (11-12/06/1999, Université de Paris V - René Descartes), Paris : L'Harmattan, pp. 133-139.

ESPILONDO Jean (1999), "Charte européenne des langues régionales ou minoritaires : la question politique", in Langues et cultures régionales de France. État des lieux, enseignement, politiques, Actes du colloque (1 1-12/06/1999, Université Paris V - René Descartes), Paris : L'Harmattan, pp. 113-118.

ÉTIENNE Bruno, GIORdAN Henri \& LAFONT Robert (1999), Le temps du pluriel : la France dans l'Europe multiculturelle, S. 1. : L'Aube, 117 p.

Euromosaic; sous la direction de Peter Nelde, Miquel Strubell, Glyn Williams (1996), Bruxelles, Luxembourg : CECA-CE-CEEA, 66 p. + tableaux.

FERrET I JACAS Joaquim (1999), "El català com a 'llengue pròpia' de les administracions públiques de Catalunya", in Estudis jurídics sobre la llei de política lingüística, 
Barcelona, Madrid : Generalitat de Catalunya, Institut d'Estudis Autonòmics, Marcial Pons, Ediciones jurídicas y sociales, S.A., pp. 107-140.

FISHMAN Joshua A. (1967), "Bilingualism with and without diglossia; diglossia with and without bilingualism", The Journal of Social Issues, Vol. XXXIII, n² 2, pp. 29-38.

FraNGi Marc (2000), "Les collectivités locales face aux langues régionales", Actualité Juridique Droit Administratif (AJDA), pp. 300-306.

GARMENDIA LASA Carmen (1998), "L'utilisation des langues régionales ou minoritaires dans la vie économique et sociale", in Conférence internationale sur la Charte européenne des langues régionales ou minoritaires, Conférence organisée par la Direction de l'environnement et des pouvoirs locaux du Conseil de l'Europe (Strasbourg, 26-27/03/1998), Strasbourg : Conseil de l'Europe, Langues régionales ou minoritaires, ${ }^{\circ} 1,1998$, pp. 41 47.

GAUTHIER Guy (2000), "La querelle de la Charte européenne des langues régionales ou minoritaires", Langues : une guerre à mort, Panoramiques, $n^{\circ} 48$, pp. 174-179.

GIORDAN Henri (1982), Démocratie culturelle et droit à la différence, Paris : La Documentation française, $108 \mathrm{p}$.

GRAU Richard (1992), "Le statut juridique des droits linguistiques en France", in Les minorités en Europe : droits linguistiques et droits de l'Homme, sous la direction d'Henri Giordan, Paris : Kimé, pp. 93-112.

Guillorel Hervé (2000), "La France et la Charte européenne des langues régionales ou minoritaires", Communication préparée dans le cadre du XVIII ème Congrès mondial de l'Association Internationale de Science Politique (1-5 août 2000), Québec : Comité de Recherches 14, Les ethnies et la politique/Politics and ethnicity, $32 \mathrm{p}$.

JONES Elin Haf Gruffydd (1998), "Moyens de communication de masse, activités culturelles et langues régionales ou minoritaires", in Conférence internationale sur la Charte européenne des langues régionales ou minoritaires, Conférence organisée par la Direction de l'environnement et des pouvoirs locaux du Conseil de l'Europe (Strasbourg, 2627/03/1998), Strasbourg : Conseil de l'Europe, Langues régionales ou minoritaires, $\mathrm{n}^{\circ} 1$, pp. 34-40.

Langues et droits : langues du droit, droit des langues, Actes du colloque (Université de Paris X - Nanterre, 22-24 octobre 1998) rassemblés par H. Guillorel et G. Koubi, Bruxelles : Bruylant, 1999, 408 p.

MARCELlesı J. B. (1988), "Korsisch : Soziolinguistik. Sociolinguistique. A lingua corsa è a so situazioni sociolinguistica", in Lexikon der Romanistischen linguistik, vol. IV, Tübingen : M. Niemeyer, pp. 809-820.

Martinet André (1969), Langue et fonction, Paris : Denoël, 221 p. [éd. originale, A Functional View of Language, Oxford : Clarendon, 1962].

MAus Didier (2000), "Charte européenne des langues régionales ou minoritaires, décision du 15 juin 1999”, Encyclopedia Universalis 2000, p. 158.

MÉlin-SouCramanien Ferdinand (1999), "La République contre Babel. À propos de la décision du Conseil Constitutionnel $n^{\circ}$ 99-412 DC du 15 juin 1999, Charte européenne des langues régionales ou minoritaires", Revue de Droit public, ${ }^{\circ}$ 4, pp. 985-1003.

Mirambell i ABANCó Antoni (1999), "El català com a 'llengua pròpia' de Catalunya", in Estudis jurídics sobre la llei de política lingüística, Barcelona, Madrid : Generalitat de Catalunya, Institut d'Estudis Autonòmics, Marcial Pons, Ediciones jurídicas y sociales. S.A., pp. 45-75.

MoutouH, Hugues (1999a), "Les langues régionales en droit français", Regards sur l'actualité, $\mathrm{n}^{\circ} 250$, avril, pp. 33-41.

Moutouн, Hugues (1999b), "Vers un statut des langues régionales en droit français ?", in Langues et droits : langues du droit, droit des langues, Actes du colloque (Université de Paris X - Nanterre, 22-24/10/1998), Bruxelles : Bruylant, pp. 221-249. 
MulJaCiC Z. (1986), "L'enseignement de Heinz Kloss (modifications, implications, perspectives)", Langages, $\mathrm{n}^{\circ}$ 84, pp. 53-63.

OYHARÇABAL Bernard (1999), "La Charte européenne des langues régionales ou minoritaires", in Langues et cultures régionales de France. État des lieux, enseignement, politiques, Actes du colloque (11-12/06/1999, Université Paris V - René Descartes), Paris : L'Harmattan, pp. 119-123.

PAGÈs Catarina (1995), "Portée et contenu de la Charte européenne", in Cinquième rencontre interrégionale Langues et cultures spécifiques (22-24 juin 1995), Ustaritz : Institut Culturel Basque, pp. 68-84.

Poignant Bernard (1998), Langues et cultures régionales. Rapport de Monsieur Bernard Poignant Maire de Quimper à Monsieur Lionel Jospin Premier Ministre, juillet, 72 p. (http://www.culture.gouv.fr/culture/dglf/, sous "Les langues de France").

Poignant Bernard (2000), Langues de France : osez l'Europe !, Montpellier : Indigène éditions, $159 \mathrm{p}$.

Puig Lluís Maria de (1995), "El Consell d'Europa i la Carta de les llengües", in Drets lingüistics $i$ drets culturals a les regions d'Europa. Actes del Simposi internacional (Girona, 2325/04/1992), Barcelona : Generalitat de Catalunya, pp. 91-94.

Rapport explicatif (1993), Charte européenne des langues régionales ou minoritaires. Rapport explicatif. Convention ouverte à la signature le 5 novembre 1992, Strasbourg : Les éditions du Conseil de l'Europe.

RENAUT Alain (2000), "Le débat français sur les langues régionales", in Comprendre les identités culturelles, sous la direction de Will Kymlicka et Sylvie Mesure, Revue de philosophie et de sciences sociales (PUF), $\mathrm{n}^{\circ} 1$, pp. 381-400.

SANMARTI ROSET Josep M. (1994), Las políticas lingüísticas y las lenguas minoritarias en el proceso de construcción de Europa, S.I. : Instituto Vasco de Administración Pública, s.d., 448 p. [pp. 183-217].

SOlÉ I DURANy Joan Ramon (1996), "El concepte de llengua pròpia en el dret i en la normalització de l'idioma a Catalunya", Revista de llengua $i$ dret, $\mathrm{n}^{\circ}$ 26, pp. 95-120.

STEWART W. A. (1968), "Sociolinguistic Typology of Multilingualism", in Readings in the Sociology of Language, ed. par J. A. Fishman, The Hague, Paris : Mouton, pp. 531-545.

TICHY Heinz (2000), Die Europäische Charta der Regional-oder Minderheitensprachen und das österreichische Recht, Klagenfurt/Celovec - Ljubljana - Wien : Mohorjeva Hermagoras, $142 \mathrm{p}$.

VERPEAUX Michel (1999), "Décision n 99-412 DC du 15 juin 1999, Charte européenne des langues régionales ou minoritaires, JO 18 juin 1999, p. 8964", Jurisprudence du Conseil Constitutionnel, pp. 594-603.

VIAUT Alain (1999), "Reconnaissance légale et représentations de la langue en situation de contact linguistique (autour des notions de loyauté et de prestige)", in Langues et droits : langues du droit, droit des langues, Actes du colloque (Université de Paris X - Nanterre, 22-24 octobre 1998), rassemblés par H. Guillorel et G. Koubi, Bruxelles : Bruylant, pp. 31-56.

WOEHRLING Jean-Marie (1992), "Institutions européennes et droits des peuples", in Les minorités en Europe : droits linguistiques et droits de l'Homme, sous la direction d'Henri Giordan, Paris : Kimé, pp. 509-521.

WOEHRLING Jean-Marie (1998), “Les problèmes relatifs à l'utilisation des langues régionales ou minoritaires devant les autorités publiques et les juridictions", in Conférence internationale sur la Charte européenne des langues régionales ou minoritaires, Conférence organisée par la Direction de l'environnement et des pouvoirs locaux du Conseil de l'Europe (Strasbourg, 26-27/03/1998), Strasbourg : Conseil de l'Europe, Langues régionales ou minoritaires, $\mathrm{n}^{\circ} 1$, pp. 24-33. 